ARTICLE

https://doi.org/10.1038/s41467-019-14245-4

\title{
Bacterial Hsp70 resolves misfolded states and accelerates productive folding of a multi-domain protein
}

\author{
Rahmi Imamoglu (1) ${ }^{1}$, David Balchin (10 ${ }^{1 \star}$, Manajit Hayer-Hartl (i] ${ }^{1 \star} \&$ F. Ulrich Hartl (D) ${ }^{1 \star}$
}

The ATP-dependent Hsp70 chaperones (DnaK in E. coli) mediate protein folding in cooperation with J proteins and nucleotide exchange factors (E. coli DnaJ and GrpE, respectively). The Hsp70 system prevents protein aggregation and increases folding yields. Whether it also enhances the rate of folding remains unclear. Here we show that DnaK/DnaJ/GrpE accelerate the folding of the multi-domain protein firefly luciferase (FLuc) 20-fold over the rate of spontaneous folding measured in the absence of aggregation. Analysis by single-pair FRET and hydrogen/deuterium exchange identified inter-domain misfolding as the cause of slow folding. DnaK binding expands the misfolded region and thereby resolves the kineticallytrapped intermediates, with folding occurring upon GrpE-mediated release. In each round of release DnaK commits a fraction of FLuc to fast folding, circumventing misfolding. We suggest that by resolving misfolding and accelerating productive folding, the bacterial Hsp70 system can maintain proteins in their native states under otherwise denaturing stress conditions.

\footnotetext{
${ }^{1}$ Max Planck Institute of Biochemistry, Department of Cellular Biochemistry, Martinsried, Germany. *email: balchin@biochem.mpg.de; mhartl@biochem.mpg.de; uhartl@biochem.mpg.de
} 
sp70 chaperones function as a central hub of the protein homeostasis network in bacteria and eukaryotic cells ${ }^{1-3}$. They recognize 5-7 amino acid sequence elements that are enriched in hydrophobic residues and are typically exposed by non-native protein substrates ${ }^{1,2}$. ATP-dependent binding and release of such segments allows Hsp70s to participate in a wide range of cellular processes, including protein folding, refolding, disaggregation, and protein transfer to cellular compartments or the proteolytic machinery ${ }^{1}$. Hsp70 proteins consist of an $\mathrm{N}$ terminal nucleotide-binding domain (NBD) of $\sim 40 \mathrm{kDa}$ and a Cterminal substrate-binding domain (SBD) of $\sim 30 \mathrm{kDa}$, connected by a hydrophobic linker. The SBD is composed of a $\beta$-sandwich domain, harboring the peptide-binding site, and an $\alpha$-helical lid segment. Peptide substrate binds in an extended conformation in a groove in the $\beta$-sandwich domain ${ }^{1,2,4}$.

The activity of Hsp70 in protein folding is regulated by Hsp40 (or J-domain) proteins and nucleotide exchange factors, which coordinate a highly allosteric reaction cycle. This reaction cycle is best understood for the E. coli system, consisting of DnaK (Hsp70), DnaJ (Hsp40) and the nucleotide exchange factor GrpE (herein referred to as KJE) (Fig. 1). DnaJ is a chaperone that functions in recognizing and transferring substrate proteins to DnaK in the ATP state ${ }^{5,6}$, in which the hydrophobic interdomain linker and the $a$-helical lid of the SBD are associated with the NBD, and the SBD is in an open conformation ${ }^{7-10}$. In this state DnaK has high on- and off-rates for substrate. Interaction of DnaJ with DnaK strongly accelerates (by $>1000$-fold) the hydrolysis of the bound ATP, generating the ADP state, in which SBD and NBD are loosely associated and the $\alpha$-helical lid is closed, trapping the bound substrate (low on- and off-rates) ${ }^{11,12}$ (Fig. 1). Subsequent binding of GrpE to the NBD facilitates ADPATP exchange, opening the SBD and allowing substrate release for folding or transfer to downstream chaperones such as the chaperonin GroEL ${ }^{6}$. Rebinding to DnaK prevents off-pathway aggregation, with successive cycles resulting in high folding yields for proteins that would aggregate in the absence of chaperones $^{5,13,14}$.

The KJE system mediates the folding of newly synthesized proteins and the refolding of proteins that unfolded under stress

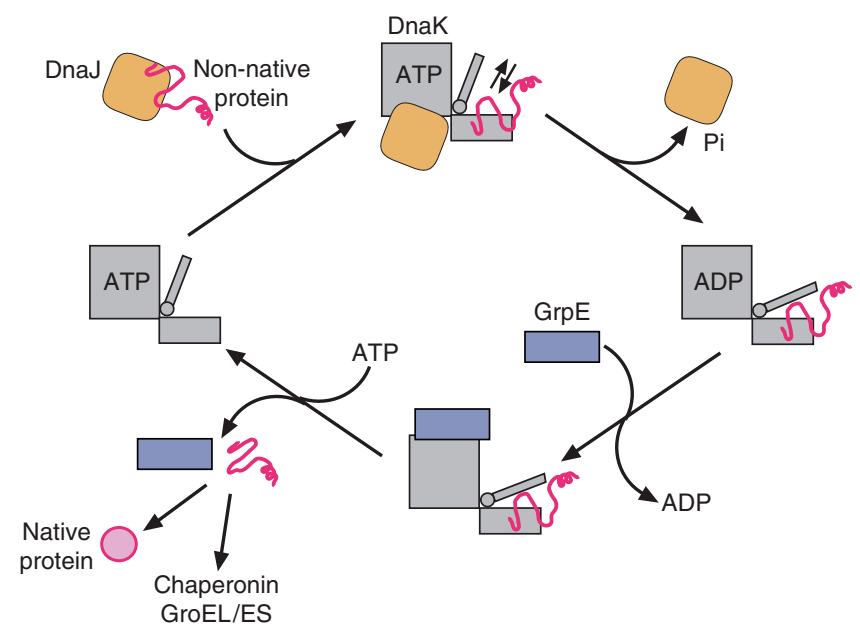

Fig. 1 Dnak/DnaJ/GrpE reaction cycle. DnaJ captures the substrate protein and transfers it to DnaK in the ATP-bound state. DnaJ and substrate synergistically trigger ATP hydrolysis by DnaK, thereby generating a stable complex between the substrate and DnaK in the ADP-bound state. Catalysis of ADP-ATP exchange by GrpE stimulates client release and regenerates DnaK-ATP for another round of client engagement. Figure modified from ref. 68 . conditions such as heat stress, where DnaK and its co-chaperones are strongly induced ${ }^{15-18}$. Among the $\sim 700$ identified E. coli substrates of DnaK are numerous multi-domain proteins, as well as proteins that need to be stabilized for subsequent interaction with GroEL ${ }^{17,19}$. In de novo protein folding DnaK cooperates with the ribosome-binding chaperone Trigger factor, which acts upstream of DnaK ${ }^{15,16,20}$.

How exactly protein binding and release by Hsp70 translates into productive folding is not yet understood. Specifically, it remains to be determined whether and how this chaperone system modulates the energy landscape of folding reactions beyond preventing off-pathway aggregation. Indeed, it has been proposed that KJE can use the energy of ATP to catalytically unfold misfolded states ${ }^{14,21,22}$, and stabilize native states out of equilibrium under denaturing conditions ${ }^{18,23}$. A key question in this context is whether KJE accelerates protein folding under conditions in which neither the folding rate nor the yield is limited by aggregation. Here we addressed this question using firefly luciferase (FLuc) as a multi-domain model protein. FLuc is thermally unstable and highly aggregation-prone during folding, and due to its sensitive luminescence assay has been a preferred Hsp70 substrate in studies in vitro and in vivo $5,13,24,25$. We used single-pair fluorescence resonance energy transfer (spFRET) to study the folding of FLuc in the absence of confounding effects due to protein aggregation. This approach revealed that the E. coli Hsp70 system substantially accelerates FLuc refolding, and enabled direct detection of both spontaneous misfolding and conformational rescue of misfolded states by the KJE chaperone machinery. Hydrogen/deuterium exchange coupled to mass spectrometry (H/DX-MS) served to localize FLuc misfolding to the subdomain interface of the large $\mathrm{N}$-terminal domain.

\section{Results}

The E. coli Hsp70 system accelerates FLuc folding. FLuc is a $\sim 60 \mathrm{kDa}$ protein consisting of a large $\mathrm{N}$-domain (residues 1-440) and a smaller C-domain (residues $441-550)^{26}$ (Fig. 2a). The Ndomain can be divided into small $\left(\mathrm{N}_{\mathrm{S}}\right.$; residues 1-190) and large $\left(\mathrm{N}_{\mathrm{L}}\right.$; residues 191-440) subdomains, of which the former has been shown to fold co-translationally ${ }^{24,27}$. DnaK/DnaJ/GrpE (KJE), as well as the eukaryotic Hsp70 chaperone system, can refold both heat- and chemically denatured FLuc $5,13,28,29$. To understand the mechanism of Hsp70-mediated folding, we monitored the rates of spontaneous and KJE-assisted folding of FLuc by sensitive luminescence assay ${ }^{30}$. Consistent with previous reports ${ }^{31,32}$, spontaneous renaturation was slow $\left(t_{1 / 2} \sim 75 \mathrm{~min}\right)$, with aggregation limiting the folding yield to $\sim 25 \%$ even at a concentration of $100 \mathrm{nM}$ FLuc (Fig. 2b and Supplementary Table 1). In contrast, KJE-assisted folding was $\sim 90 \%$ efficient and $\sim 18$-fold faster $\left(t_{1 / 2} \sim 4 \mathrm{~min}\right)$.

To exclude aggregation as the cause of slow folding, we established the critical concentration for FLuc aggregation. Dualcolor fluorescence cross-correlation spectroscopy (dcFCCS) showed absence of aggregation during refolding for equimolar mixtures of the labelled proteins at a total concentration below 10 nM (Supplementary Fig. 1a). Double-labelled FLuc (5 nM) served as positive control. Reducing the concentration of FLuc during refolding increased the yield of spontaneous folding up to $\sim 60 \%$ (Fig. $2 \mathrm{c}$ and Supplementary Table 1). The folding rate remained unchanged, indicating that aggregation was not the cause but rather the consequence of slow folding. Note that the non-ionic detergent Tween 20 (T20; 0.05\%) was used to prevent FLuc adsorption to tube walls during folding (Supplementary Fig. 1b, c). Refolding yield and kinetics were independent of the presence or absence of ATP (Supplementary Fig. 1d). The limited yield of renaturation indicated that a fraction of FLuc populates kinetically trapped folding intermediate(s) that do not convert 

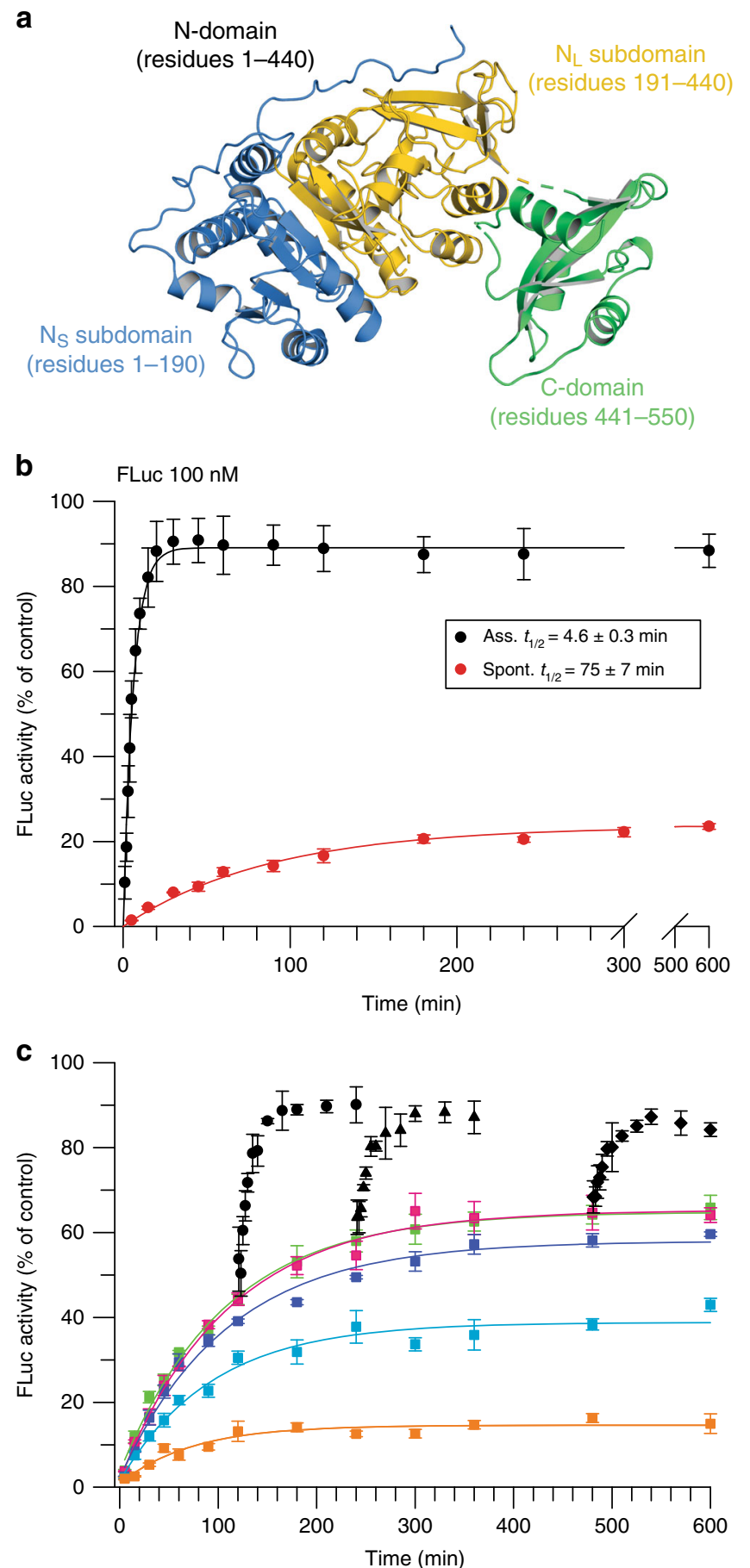

\begin{tabular}{|l|}
\hline - Spont. $0.1 \mathrm{nM}$ \\
- Spont. $1 \mathrm{nM}$ \\
- Spont. $10 \mathrm{nM}$ \\
- Spont. $50 \mathrm{nM}$ \\
- Spont. $200 \mathrm{nM}$ \\
- Spont. $1 \mathrm{nM}+$ KJE-ATP $2 \mathrm{~h}$ \\
- Spont. $1 \mathrm{nM}$ + KJE-ATP $4 \mathrm{~h}$ \\
\end{tabular}

to the native state within the time frame of our experiments $(10 \mathrm{~h})$.

To test whether kinetically trapped intermediates of FLuc (in the absence of aggregation at $1 \mathrm{nM}$ FLuc) remained competent for refolding by KJE, we transferred aliquots of the reaction after
Fig. 2 The KJE chaperone system accelerates FLuc folding. a Structure of FLuc (PDB ID: $1 \mathrm{LCl}$ ) with domains indicated. b Spontaneous and chaperoneassisted folding of $100 \mathrm{nM}$ FLuc was assayed upon dilution from $5 \mathrm{M} \mathrm{GuHCl}$ into buffer without or with KJE-ATP, respectively, by monitoring luminescence activity. KJE-mediated folding was performed with $3 \mu \mathrm{M} \mathrm{DnaK}$, $1 \mu \mathrm{M}$ DnaJ, $1.5 \mu \mathrm{M}$ GrpE and $5 \mathrm{mM}$ ATP. Error bars represent s.d. $(n=10)$. c Concentration dependence of FLuc refolding. Fluc was diluted from denaturant to different final concentrations (0.1-200 nM) and refolding assayed as in (b). Rescue of spontaneous folding reactions was assayed by adding KJE-ATP (0.3 $\mu \mathrm{M}$ DnaK, $0.1 \mu \mathrm{M}$ DnaJ, $0.5 \mu \mathrm{M}$ GrpE and $5 \mathrm{mM}$ ATP) after 2,4 or $8 \mathrm{~h}$. Error bars represent s.d. $(n=3)$. Ass., KJE-ATP-assisted; Spont., spontaneous folding. Source data are provided as a Source Data file.

different times of spontaneous folding (up to $8 \mathrm{~h}$ ) into fresh tubes containing KJE-ATP. This resulted in accelerated folding to full yield (Fig. 2c). Without ATP, refolding in the presence of KJE occurred with a rate similar to that of the spontaneous reaction, but reached a yield of only $\sim 40 \%$ (Supplementary Fig. 1e), apparently due to the binding of FLuc folding intermediate to DnaJ/DnaK. Addition of ATP resulted in accelerated folding to full yield (Supplementary Fig. 1e), consistent with the proposal that KJE can use the energy of ATP hydrolysis to shift the folding reaction out of equilibrium towards the native state ${ }^{23}$.

Together these findings demonstrate that the Hsp70 chaperone system actively promotes client protein folding in an ATPdependent manner, by reversing and/or averting misfolding.

Slow folding of FLuc is caused by inter-domain misfolding. To understand the basis of the slow and inefficient spontaneous folding of FLuc, we next monitored folding by spFRET combined with pulsed interleaved excitation (PIE) ${ }^{33}$. The spFRET experiments were performed at $50 \mathrm{pM}$ FLuc, at which concentration FLuc is monomeric during refolding as measured by dcFCCS (Supplementary Fig. 1a). We designed four double-cysteine mutants of FLuc and labelled them with Atto532 and Atto647N as fluorescence donor and acceptor, respectively $\left(R_{\mathrm{o}} \sim 46 \AA\right)^{34}$. Note that fluorophore attachment preserved FLuc foldability and activity (Supplementary Fig. 2a-d). FLucNC (D19C/S504C) reports on overall FLuc folding, FLucN (D19C/E428C) on folding of the N-domain, FLucN $\mathrm{S}_{\mathrm{S}}$ (D19C/S170C) on the $\mathrm{N}_{\mathrm{S}}$-subdomain, and FLucC (D476C/S504C) on folding of the C-domain (Fig. 3a). We observed symmetric distributions of FRET efficiencies $\left(f_{\mathrm{E}}\right)$ for each construct, with mean $f_{\mathrm{E}}$ values in good agreement with fluorophore distances predicted from the crystal structure (Fig. 3b-e). Upon dilution from denaturant ( $0 \mathrm{~h}$ time point), the FLucNC, FLucN and FLucN $\mathrm{N}_{\mathrm{S}}$ proteins converted from the low $f_{\mathrm{E}}$ state of the denatured protein to a broad $f_{\mathrm{E}}$ distribution (Fig. 3f-h). These distributions include conformations that are substantially more compact than the native state and thus are likely to contain non-native interactions (denoted as misfolded; $\mathrm{MF}$ ), consistent with the detection of misfolded states of FLuc in optical tweezer experiments ${ }^{35}$. During folding these intermediates shifted towards the $f_{\mathrm{E}}$ distribution of the native state (Fig. $3 \mathrm{f}-\mathrm{h}$ ) with an apparent $t_{1 / 2}$ of $\sim 70-85$ min (Supplementary Fig. 2e-g). In contrast, FLucC adopted the native state $f_{\mathrm{E}}$ at least 10 -fold faster without populating detectable intermediates (Fig. 3e, i and Supplementary Fig. 2h), suggesting that the C-domain folds independently of the $\mathrm{N}$-domain. Interestingly, rapid folding was also observed for the isolated $\mathrm{N}_{\mathrm{S}}$-subdomain (FLuc190) (Fig. 3j, $\mathrm{k})$, consistent with its ability to fold co-translationally ${ }^{27,36}$. This is in contrast to the slow folding of $\mathrm{N}_{\mathrm{S}}$ in the context of full-length FLuc $\left(\mathrm{FLucN}_{\mathrm{S}}\right)$, which limits the folding yield (Fig. $3 \mathrm{~h}, \mathrm{k}$ and Supplementary Fig. 2g). Thus, folding of the N-domain is the 


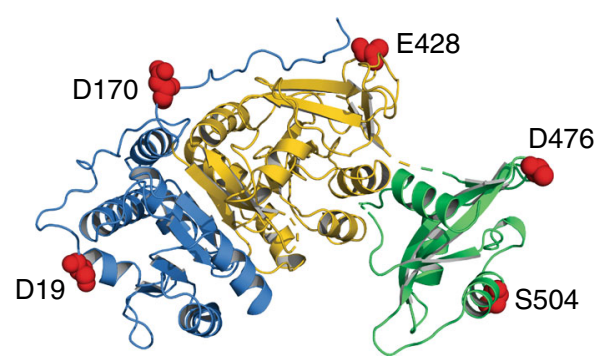

Distance $(\AA)$

66.8

37.1

35.4

24.4

35.4
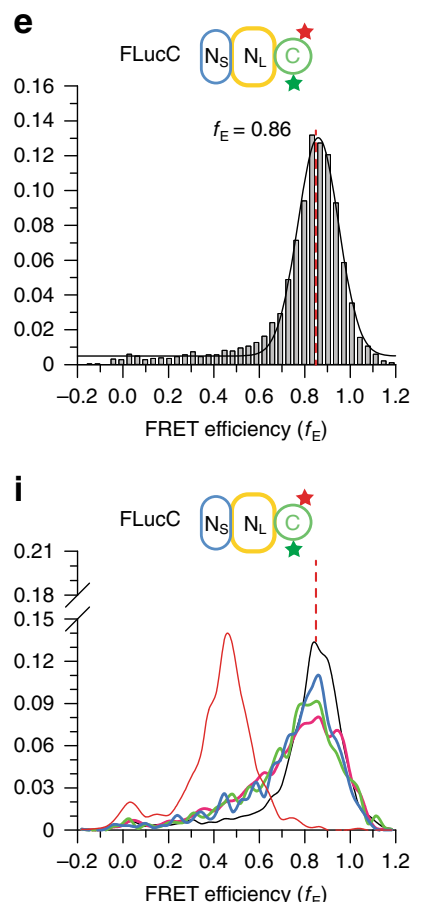

b

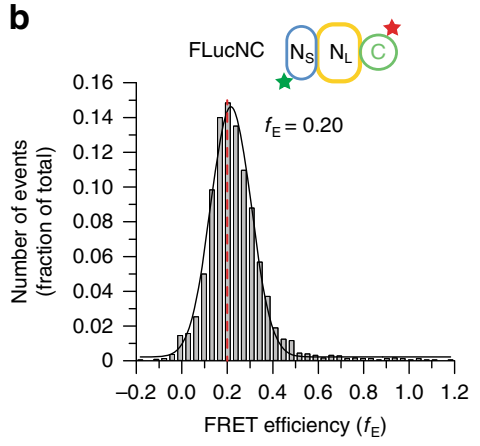

f

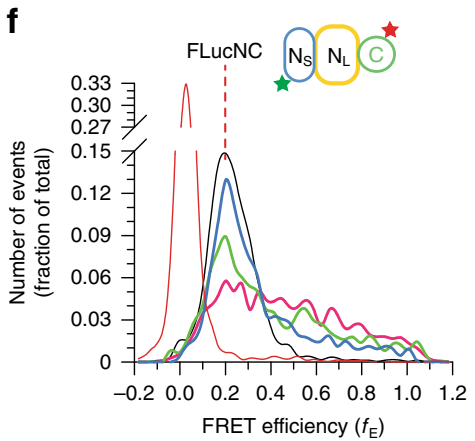

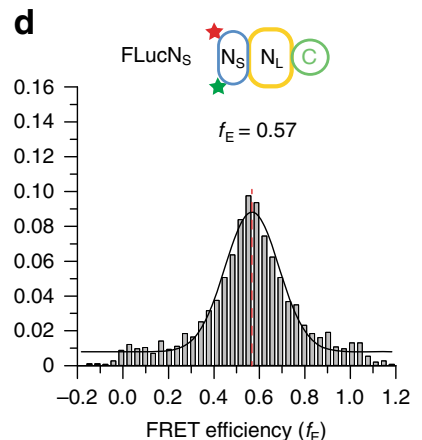

h

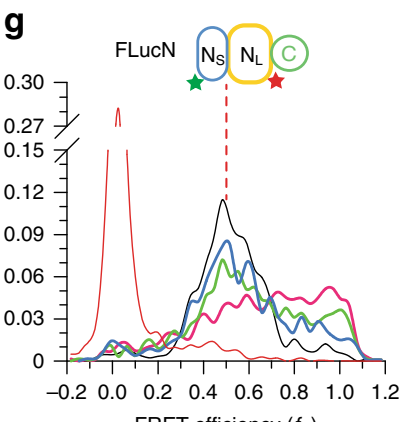

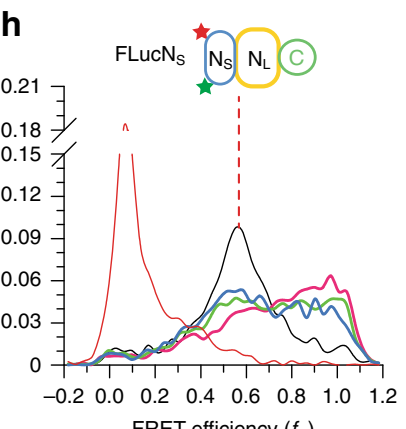

FRET efficiency $\left(f_{\mathrm{E}}\right)$

- Native - Spont. $0 \mathrm{~h}-$ Spont. $3 \mathrm{~h}-$ Spont. $9 \mathrm{~h}-5 \mathrm{M} \mathrm{GuHCl}$
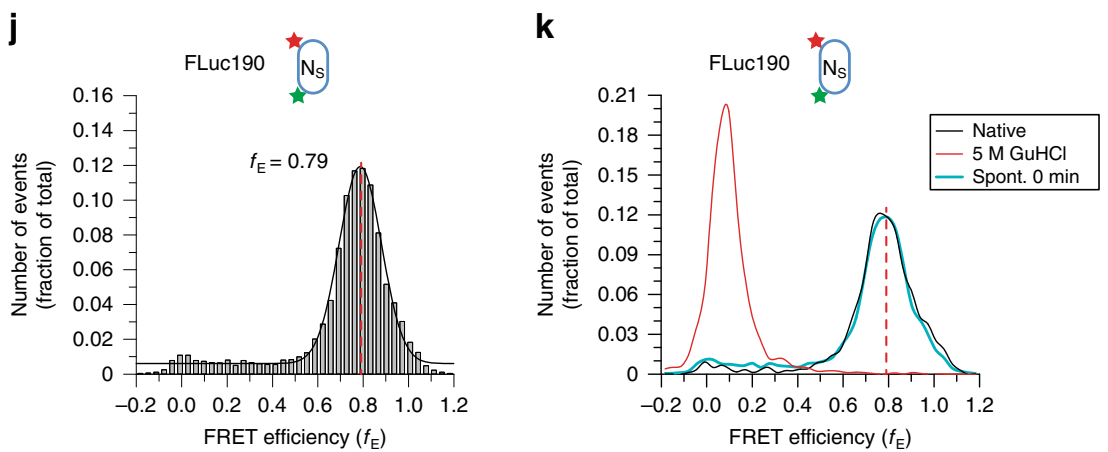

Fig. 3 Slow spontaneous folding of FLuc is caused by inter-domain misfolding. a Structure of FLuC (PDB ID: 1LCI) showing fluorophore labelling positions. Mutations introduced to allow site-specific cysteine labelling are indicated, and estimated distances between dye pairs are given. FLuc variants were labelled with Atto532 (donor) and Atto647N (acceptor). b-e spFRET efficiency ( $f_{E}$ ) histograms of the native proteins: FLucNC (b), FLucN (c), FLucN $($ d) and FLuCC (e). $\mathbf{f}-\mathbf{i}, f_{\mathrm{E}}$ histograms measured during spontaneous refolding of $50 \mathrm{pM}$ denatured FLuc. spFRET was recorded for $1 \mathrm{~h}$, either immediately ( $0 \mathrm{~h}$ ) or at the indicated folding time points. $\mathbf{j}$, k, spFRET efficiency $\left(f_{\mathrm{E}}\right)$ histogram of the $\mathrm{N}_{\mathrm{S}}$-subdomain (FLuc190) (j) and spontaneous folding of $50 \mathrm{pM}$ denatured FLuc190 (k). Data were recorded for 30 min. Reactions b-k contained $5 \mathrm{mM} \mathrm{ATP}$ and $50 \mu \mathrm{M}$ phenobenzothiazine (PBT). The $f_{\mathrm{E}}$ corresponding to the native state for each construct is indicated by a dashed vertical red line. Representative measurements of three independent repeats are shown. Spont., spontaneous folding. Source data are provided as a Source Data file. 

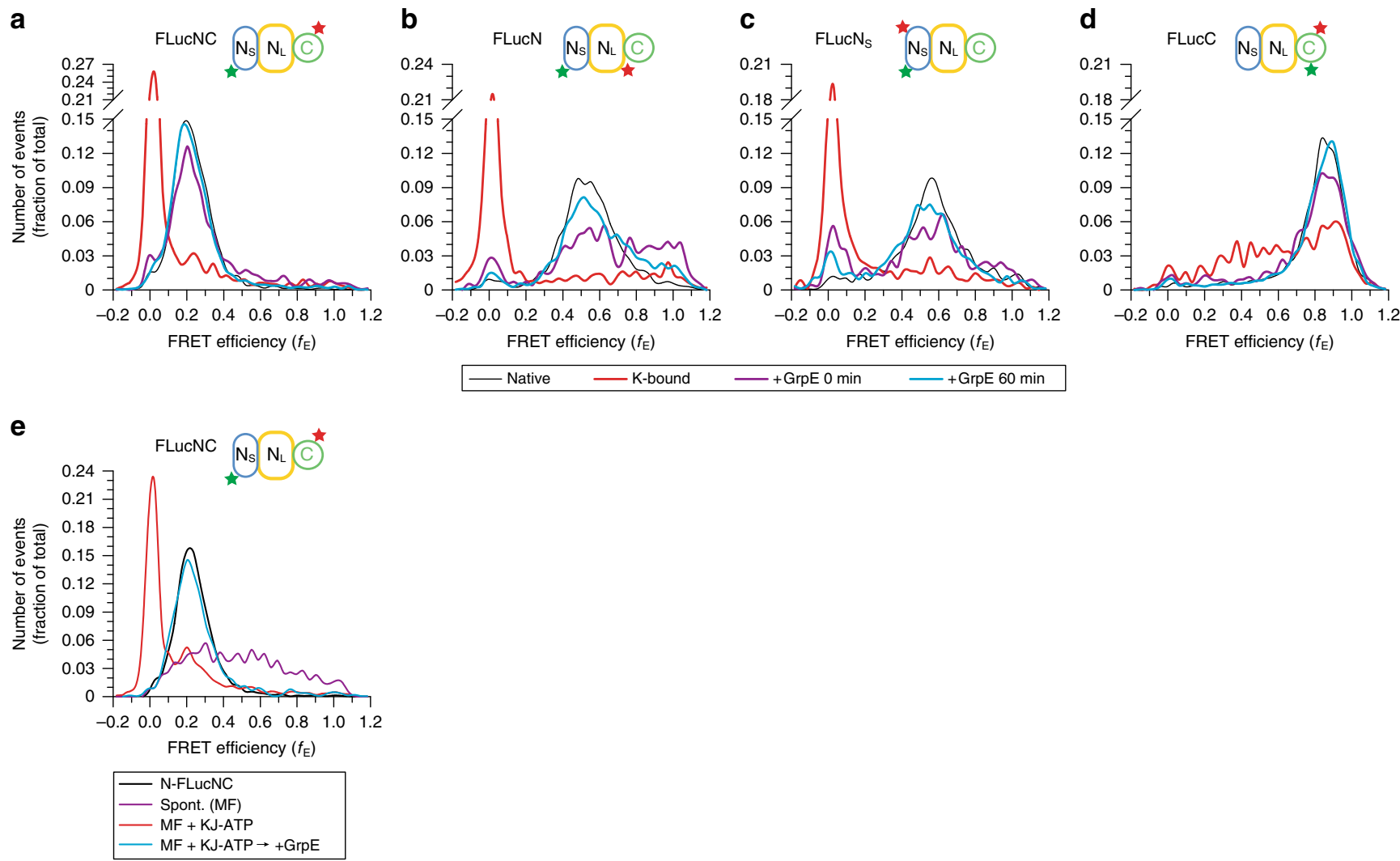

Fig. 4 KJE reverses misfolding and commits bound protein to fast folding. a-d spFRET efficiency histograms measured during KJE-ATP assisted folding of FLucNC (a), FLucN (b), FLucN $(\mathbf{c}$ ) or FLucC (d). GuHCl-denatured FLuc was diluted to $50 \mathrm{pM}$ in buffer containing $0.3 \mu \mathrm{M}$ DnaK, $0.1 \mu \mathrm{M}$ DnaJ, $5 \mathrm{mM}$ ATP and $50 \mu \mathrm{M}$ PBT, and the $f_{\mathrm{E}}$ distribution of DnaK-bound FLuc was recorded, followed by initiation of folding with $0.5 \mu \mathrm{M}$ GrpE. spFRET was recorded for $15 \mathrm{~min}$ either immediately $(0 \mathrm{~min})$ or after $60 \mathrm{~min}$ of folding. Representative measurements of three independent repeats are shown. e KJ-ATP converts compact, misfolded (MF) intermediates of FLuc to the DnaK-bound, expanded state. GuHCl-denatured FLucNC was diluted to 50 pM in buffer containing ATP and PBT, and the distance between $\mathrm{N}$ - and C-domains probed by spFRET as in (a). KJ-ATP were added after $1 \mathrm{~h}$ to generate DnaK-bound FLuc, followed by GrpE to initiate folding. Representative measurements of three independent repeats are shown. Spont., spontaneous folding. Source data are provided as a Source Data file.

rate-limiting step during spontaneous folding and involves misfolding between $\mathrm{N}_{S}$ and $\mathrm{N}_{\mathrm{L}}$.

KJE-assisted folding involves unfolding of misfolded states. How does the KJE chaperone system accelerate FLuc folding? To characterize the state of FLuc from which chaperone-assisted folding initiates, we diluted denatured FLuc into folding buffer containing DnaK, DnaJ and ATP (KJ-ATP). GrpE was omitted to prevent substrate release ${ }^{5,6}$. DnaJ allows for efficient transfer of protein substrate to DnaK and dissociates upon ATP hydrolysis, resulting in a stable DnaK-substrate complex with DnaK in the ADP state ${ }^{2}$ (Fig. 1). DnaK-bound FLucNC, FLucN and FLucN exhibited very low $f_{\mathrm{E}}$ distributions similar to those of the denatured proteins, indicative of highly expanded conformations (Fig. $4 \mathrm{a}-\mathrm{c}$ ). As expected, this effect required the function of DnaJ (Supplementary Fig. 3a). In contrast, the chaperones failed to stabilize FLucC in the expanded state (Fig. 4d), consistent with its rapid folding (Fig. 3i and Supplementary Fig. 2h). Importantly, the isolated $\mathrm{N}_{\mathrm{S}}$-subdomain (FLuc190) also failed to interact with KJ-ATP (Supplementary Fig. 3b). Thus misfolding of $\mathrm{N}_{\mathrm{S}}$ in the context of the complete $\mathrm{N}$-domain results in exposure of DnaK binding sites, thereby allowing conformational expansion of the $\mathrm{N}_{\mathrm{S}}$ subdomain by DnaK.

Analytical ultracentrifugation determined a mass of $\sim 900 \mathrm{kDa}$ for the DnaK-FLuc complex (Supplementary Fig. 4a), consistent with a diffusion coefficient of $21 \mu \mathrm{m}^{2} \mathrm{~s}^{-1}$ by FCS (Supplementary Fig. $4 \mathrm{~b}$ ). dcFCCS measurements using an equimolar mixture of FLuc-
Alexa532 and FLuc-Alexa532 demonstrated that the DnaK-FLuc complex contains only one molecule of FLuc (Supplementary Fig. 4c). Moreover, dcFCCS with FLuc-Alexa532 and DnaJAlexa647 showed no significant cross-correlation, indicating that the DnaK-FLuc complex contains essentially no DnaJ $(<5 \%$ of complexes) (Supplementary Fig. 4c). The mass of $\sim 900 \mathrm{kDa}$ for the complex would correspond to $\sim 12$ DnaK bound per expanded FLuc molecule. This is consistent with the number of predicted DnaK binding motifs in FLuc (13 sites, Supplementary Fig. 4d, e) ${ }^{37}$. However, as DnaK can bind the flexible linker between the ATPase and peptide-binding domains of another $\mathrm{DnaK}^{38}$, not all DnaK molecules may be bound directly to FLuc. Folding of FLuc by the KJE system thus initiates from an unfolded state that resembles the denatured protein in terms of conformational expansion.

Addition of GrpE to the DnaK-bound FLuc resulted in a shift to the native spFRET distribution of FLuc (Fig. 4a-d). A minor population of expanded FLuc is apparent for FLucNC, FLucN and $\mathrm{FLucN}_{\mathrm{S}}$ at early time points of the reaction, suggesting that non-native FLuc transiently re-engages the chaperone system during folding (Fig. $4 \mathrm{a}-\mathrm{c}$ ). Note that KJ-ATP do not bind native FLuc (N-FLuc) (Supplementary Fig. 5a). During assisted folding, FLuc also transiently populated compact intermediates similar to the MF states observed during spontaneous folding (see Fig. 4b). To understand the fate of these intermediates during KJE cycling, we first generated compact folding intermediates of FLucNC in the absence of chaperones, and then added KJ-ATP (Fig. 4e). The broad $f_{\mathrm{E}}$ of the folding intermediates shifted to the narrow, low $f_{\mathrm{E}}$ 
a

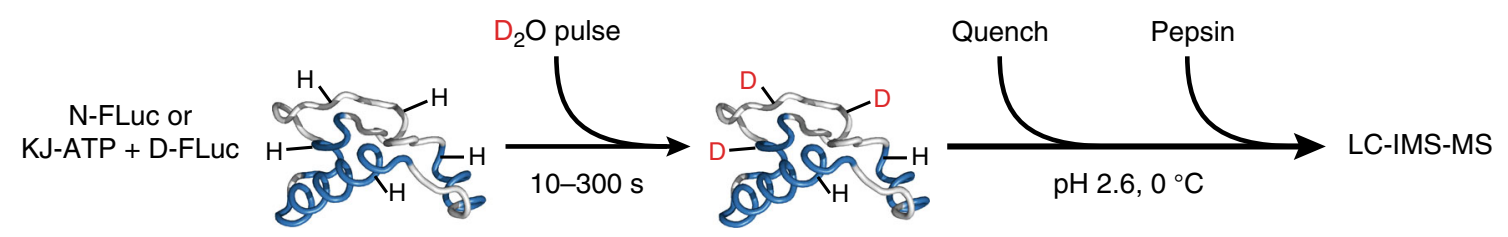

b N-FLuc

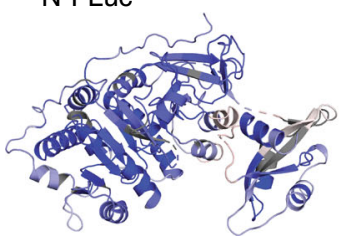

$10 \mathrm{~s}$

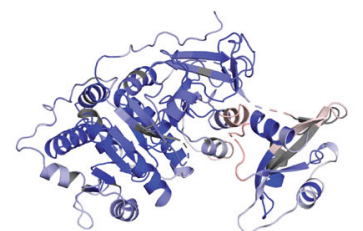

$30 \mathrm{~s}$

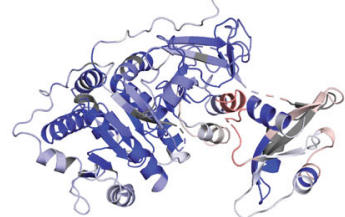

$100 \mathrm{~s}$

$$
\text { Low Heuteration }
$$

\section{K-bound}

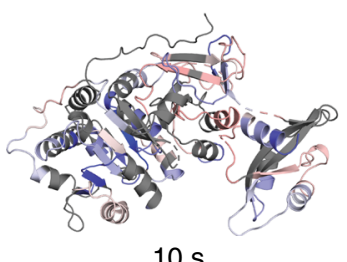

$10 \mathrm{~s}$

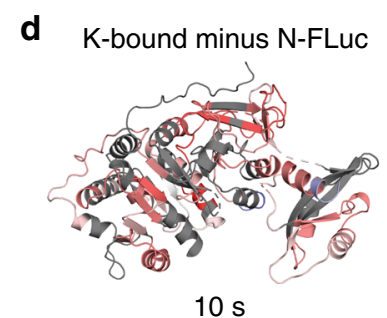

$10 \mathrm{~s}$

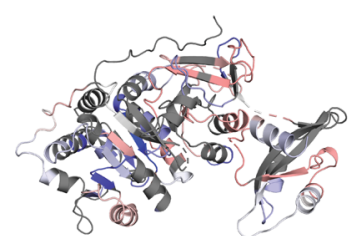

$30 \mathrm{~s}$

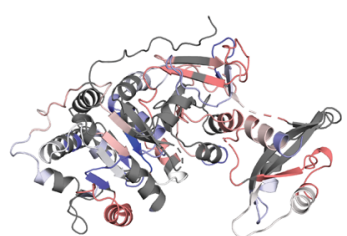

$100 \mathrm{~s}$

Low High

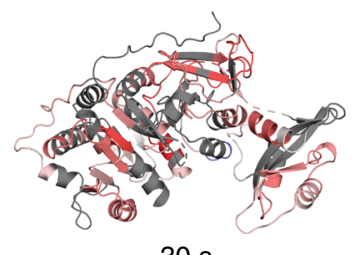

$30 \mathrm{~s}$

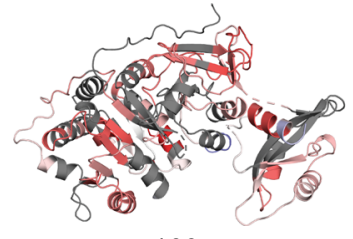

$100 \mathrm{~s}$

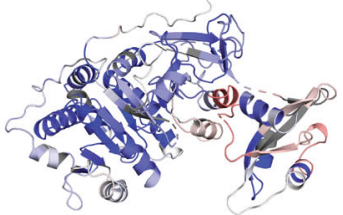

$300 \mathrm{~s}$

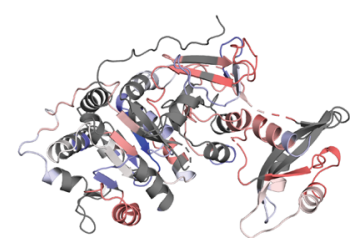

$300 \mathrm{~s}$

Protected

Deprotected

$\mathrm{H} / \mathrm{DX}$ relative to native

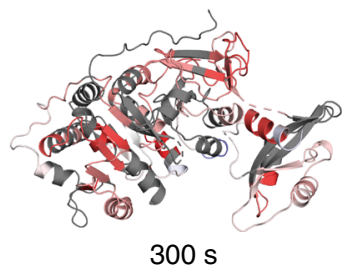

Fig. 5 Conformational dynamics of native and DnaK-bound FLuc. a Scheme of equilibrium H/DX-MS experiment. b-c, Peptide-level deuterium exchange of native FLuc (N-FLuc) (b) or DnaK-bound FLuc (K-bound) (c) after exposure to deuterium for 10-300 s. Relative fractional deuterium exchange for each peptide is mapped onto the structure of FLuc (PDB ID: $1 \mathrm{LCl}$ ) as a gradient from blue (0\%) to red (75\%). d Difference in deuterium exchange between DnaK-bound and N-FLuc at peptide level. Deuteration differences are scaled from blue $(-50 \%)$ to red $(+50 \%)$. Regions coloured red are deprotected when bound to DnaK. H/DX data are the average of three independent repeats.

characteristic of the DnaK-bound state (Fig. 4e). Note that the small amount of spontaneously folded FLucNC did not shift (Fig. 4e), as KJ-ATP does not bind N-FLuc (Supplementary Fig. 5a). Subsequent addition of GrpE resulted in efficient folding from the expanded state (Fig. 4e). A broad conformational distribution of folding intermediates (including MF states) also formed during spontaneous folding of FLuc at $37{ }^{\circ} \mathrm{C}$, followed by expansion upon chaperone-binding (Supplementary Fig. 5b). Together these data show that the mechanism of KJE-assisted folding involves unfolding of kinetically trapped misfolded states.

FLuc misfolding involves the interface of $N_{S}$ and $N_{L}$ subdomains. To extend our insight into FLuc folding from domain to peptide level, we analysed KJE-assisted folding reactions using H/DX-MS ${ }^{39,40}$. Note that H/DX-MS of spontaneous folding was not possible, because FLuc aggregates at the higher concentrations $(1 \mu \mathrm{M})$ required for these experiments. KJE-ATP-dependent refolding (10 $\mu \mathrm{M}$ DnaK/3.3 $\mu \mathrm{M}$ DnaJ/5 $\mu \mathrm{M}$ GrpE) occurred with a yield of $\sim 76 \%$ and a rate comparable to that measured at lower FLuc concentration (Supplementary Fig. 6). We first compared the conformational properties of DnaK-bound and N-FLuc by $\mathrm{D}_{2} \mathrm{O}$ pulse analysis at equilibrium (Fig. 5a). Peptide coverage was $\sim 95 \%$ for N-FLuc and $\sim 63 \%$ for the DnaK-bound protein (Supplementary Fig. 7a, b and Supplementary Data 1a). DnaK-bound FLuc was globally destabilized relative to the native protein (Fig. 5b-d and Supplementary Data 1b). The majority of peptides exchanged in the EX2 regime, and several peptides showed evidence of EX1 or mixed EX1/EX2 exchange kinetics in the DnaKbound state (Supplementary Fig. $8 \mathrm{a}-\mathrm{c}$ ). Correlated exchange at the EX1 limit is evidence of cooperative local unfolding ${ }^{40}$. The observation that parts of DnaK-bound FLuc sample both unfolded and partially folded states is consistent with the detection by nuclear magnetic resonance (NMR) of transient secondary structure in a small protein domain bound to $\mathrm{DnaK}^{41}$.

We next probed the conformation of FLuc by $10 \mathrm{~s}_{2} \mathrm{O}$ pulses at $10 \mathrm{~s}, 5 \mathrm{~min}$ and $30 \mathrm{~min}$ after initiating folding with GrpE (Fig. 6a). Consistent with the folding kinetics measured by 
a

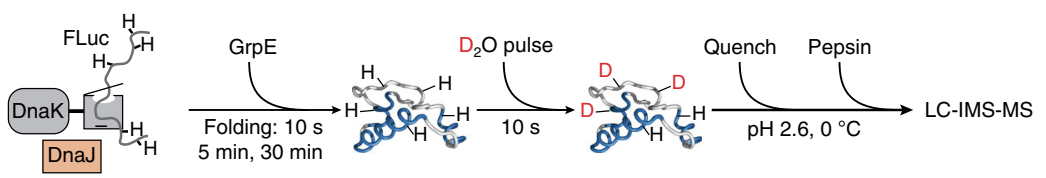

b
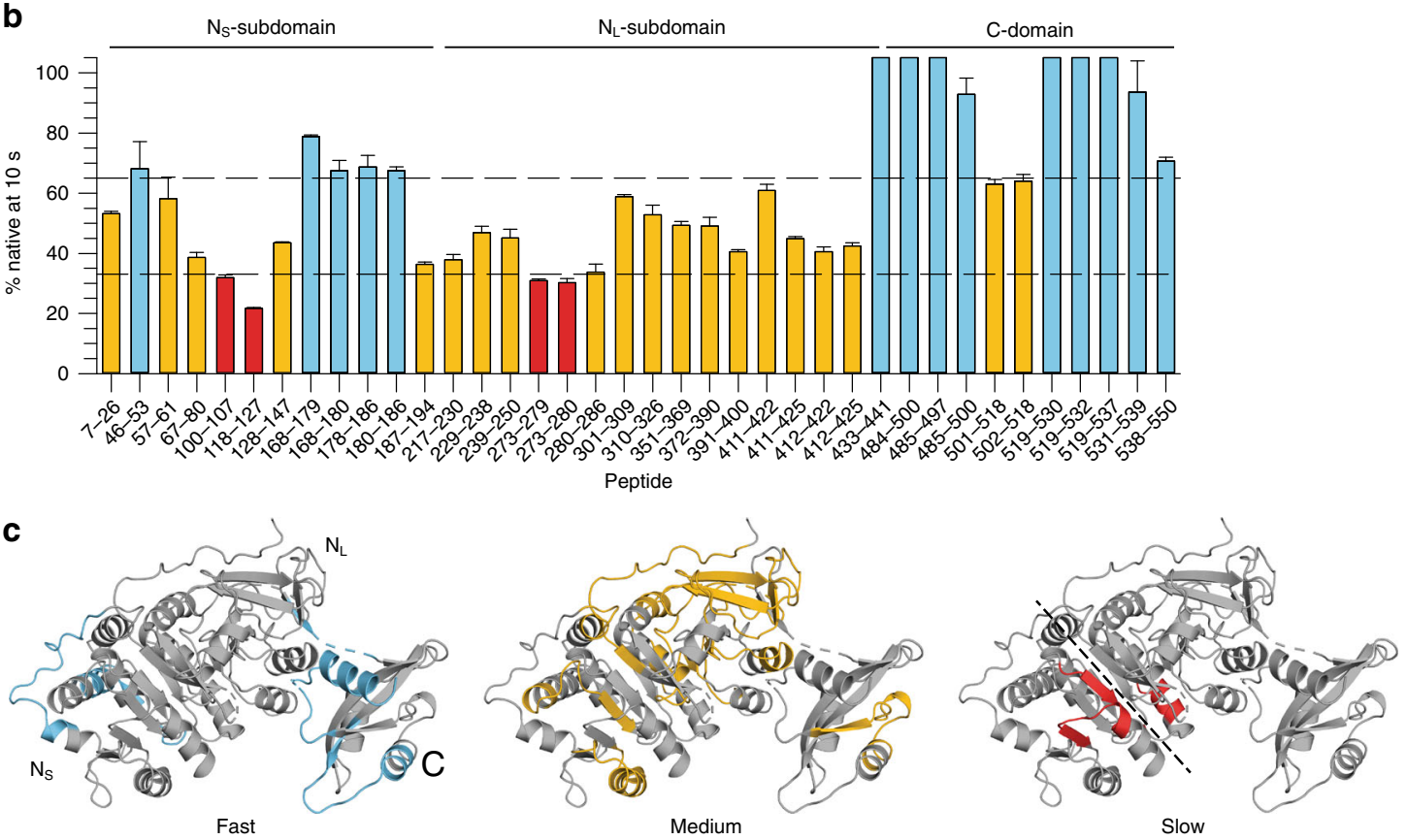

Fig. 6 KJE-assisted folding of FLuc at peptide resolution. a Scheme of H/DX-MS experiment. DnaK is shown schematically in grey and DnaJ in orange. b Fraction of native deuterium exchange reached by FLuc peptides after $10 \mathrm{~s}$ folding. D uptake at $10 \mathrm{~s}$ was normalized to the difference in $D$ uptake between K-bound and N-FLuc for each peptide. Only peptides exhibiting a >1 Da difference between the DnaK-bound and native states are shown. Peptides are characterized as fast (upper third, blue), medium (middle third, gold) or slow (lower third, red) folders, based on their relative protection compared to NFLuc. Error bars represent s.d. $(n=3)$. Source data are provided as a Source Data file. $\mathbf{c}$ Location of the peptides colour-coded as in (b), indicated on the structure of Fluc. Dotted line indicates the $\mathrm{N}_{S}-\mathrm{N}_{\mathrm{L}}$ domain interface.

luminescence assay, all peptides analysed (Supplementary Fig. 7c) acquired native protection levels within 30 min (Supplementary Data 1c). Bimodal peptide-mass distributions indicative of co-existing native and non-native populations were observed at intermediate folding times (Supplementary Fig. 9). Analysis of H/ DX protection after $10 \mathrm{~s}$ revealed that the C-domain and part of the $\mathrm{N}_{\mathrm{S}}$-subdomain (residues $46-53$ and 168-186) acquire nativelike protection rapidly, followed by the remainder of the $\mathrm{N}$ domain (Fig. 6b, c). Strikingly, the peptides that acquire protection at the slowest rate (residues 100-107, 118-127 and 273-280) are located at the interface between the $\mathrm{N}_{\mathrm{S}^{-}}$and $\mathrm{N}_{\mathrm{L}^{-}}$ subdomains (Fig. 6b, c). Formation of this interface is therefore rate limiting for overall folding, with misfolding apparently resulting in compact states of FLuc that must be resolved by a further round of chaperone action (Fig. 4e). Indeed, predicted DnaK binding motifs on FLuc are enriched at the $\mathrm{N}_{\mathrm{S}}-\mathrm{N}_{\mathrm{L}}$ interface (5 of 13 high confidence sites, Supplementary Fig. 4d, e), suggesting that non-native conformations that expose this region are efficiently targeted by DnaK.

DnaK commits a fraction of bound protein to fast folding. To understand the mechanism of accelerated folding by the KJE system, it was of interest to measure the yield and rate of folding for a single round of DnaK binding and release. We addressed this question by inhibiting the rebinding of FLuc to DnaK after GrpE-mediated release. This was achieved by addition of the peptide GSGNRLLLTG (NR) that competes for the substratebinding site on DnaK ${ }^{4,42,43}$. When excess NR was added to KJ-
ATP before denatured FLuc, folding occurred at the slow rate of the spontaneous reaction (Fig. 7), indicating inhibition of DnaK binding. Note that the reduced yield of folding $(\sim 40 \%)$ is due to the presence of DnaJ, which is not efficiently blocked by NR (see also Supplementary Fig. 1e). Addition of NR after 5 min of KJEassisted folding caused an immediate shift of the accelerated folding reaction to the slow kinetics of spontaneous folding (Fig. 7). To assess the folding reaction associated with a single chaperone cycle, we added NR to a pre-formed DnaK-FLuc complex, and then initiated chaperone release with GrpE. This resulted in biphasic folding kinetics, with a fast phase $\left(t_{1 / 2}=4 \pm 1\right.$ $\mathrm{min}$ ) identical to the folding rate in the cycling KJE reaction, and a slow phase $\left(t_{1 / 2}=49 \pm 8 \mathrm{~min}\right)$ close to the rate of spontaneous folding (Fig. 7). Approximately 15\% of FLuc reached native state during the fast phase, compared to only $\sim 3 \%$ in the equivalent time of spontaneous refolding (Fig. 7). Thus, the Hsp70 mechanism provides access to a fast folding trajectory for a fraction of FLuc molecules in each chaperone cycle, perhaps favoring a mechanism in which segments of the $\mathrm{N}_{\mathrm{S}}$-subdomain are released for fast folding (Fig. $6 b$ ), while $\mathrm{N}_{\mathrm{S}}-\mathrm{N}_{\mathrm{L}}$ interface regions remain DnaK-bound. These molecules apparently by-pass the non-native interactions within the $\mathrm{N}$-domain that are ratelimiting for spontaneous folding and are committed to fold rapidly to the native state.

KJE shifts the folding equilibrium towards the native state. To test whether accelerated folding by KJE-ATP may be important in stabilizing proteins under conditions of heat stress, we took 


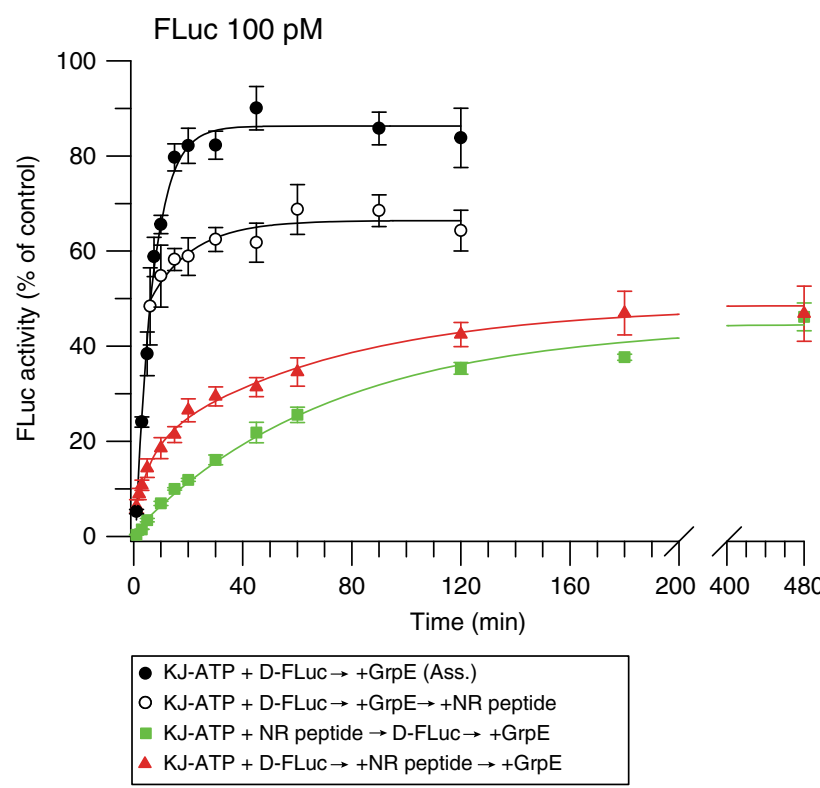

Fig. 7 DnaK commits a fraction of bound FLuc to fast folding. Folding of FLuc in a single-round of chaperone action. Rebinding of FLuc to DnaK was inhibited using excess NR peptide $(125 \mu \mathrm{M})$. For single round folding, $\mathrm{GuHCl}$-denatured FLuc was diluted to $100 \mathrm{pM}$ in buffer containing KJ-ATP, followed by addition of NR and initiation of folding with GrpE (red triangles). As controls, NR was either omitted (black circles) or added to KJ-ATP before FLuc (green squares), or after 5 min of chaperone-assisted folding (white circles). Error bars represent s.d. $(n=3)$. Ass., KJE-ATPassisted. Source data are provided as a Source Data file. advantage of the limited thermal stability of FLuc ${ }^{13,44}$. Incubation of $\mathrm{N}$-FLuc at $37^{\circ} \mathrm{C}$ resulted in loss of activity over time (Fig. 8a). Note that aggregation was avoided by using a very low FLuc concentration (10 pM) (Supplementary Fig. 10). Spontaneous folding at $37^{\circ} \mathrm{C}$ was slow and inefficient, with a final yield of only $\sim 15 \%$ (Fig. $8 \mathrm{a}$ ). In contrast, KJE-assisted folding reached $\sim 85 \%$ $\left(t_{1 / 2}=2.9 \pm 0.3 \mathrm{~min}\right)$. Addition of KJE-ATP to the spontaneous folding reaction or thermally denatured FLuc rapidly restored full activity (Fig. 8a), similar to the observations at $25^{\circ} \mathrm{C}$ (Fig. $2 \mathrm{c}$ and Supplementary Fig. 1e). When N- FLuc was incubated at $37^{\circ} \mathrm{C}$ together with KJE-ATP, activity was maintained (Fig. 8a). Upon thermal unfolding in the absence of chaperones, FLucNC populated a broad conformational distribution of folding intermediates by spFRET (Fig. 8b). In contrast, in the presence of KJ-ATP, the $\mathrm{f}_{\mathrm{E}}$ distribution of native FLucNC (N-FLucNC) gradually shifted to that of the expanded DnaK-bound state, from which it refolded upon addition of GrpE (Fig. 8b).

These results demonstrate the ability of the KJE chaperone system to use the energy of ATP to shift the folding equilibrium of proteins like FLuc towards the native state under denaturing conditions, consistent with recent observations ${ }^{18,23}$. Our findings indicate that KJE performs this function by unfolding kinetically trapped, misfolded states and allowing re-folding to the native state along a fast trajectory that circumvents misfolding.

\section{Discussion}

In the canonical model of Hsp70 function, nucleotide-driven cycles of binding and release inhibit aggregation of the client protein without influencing its folding pathway. Here we demonstrated an additional function of Hsp70 in accelerating the folding of a model multi-domain protein and analysed its mechanism. During a

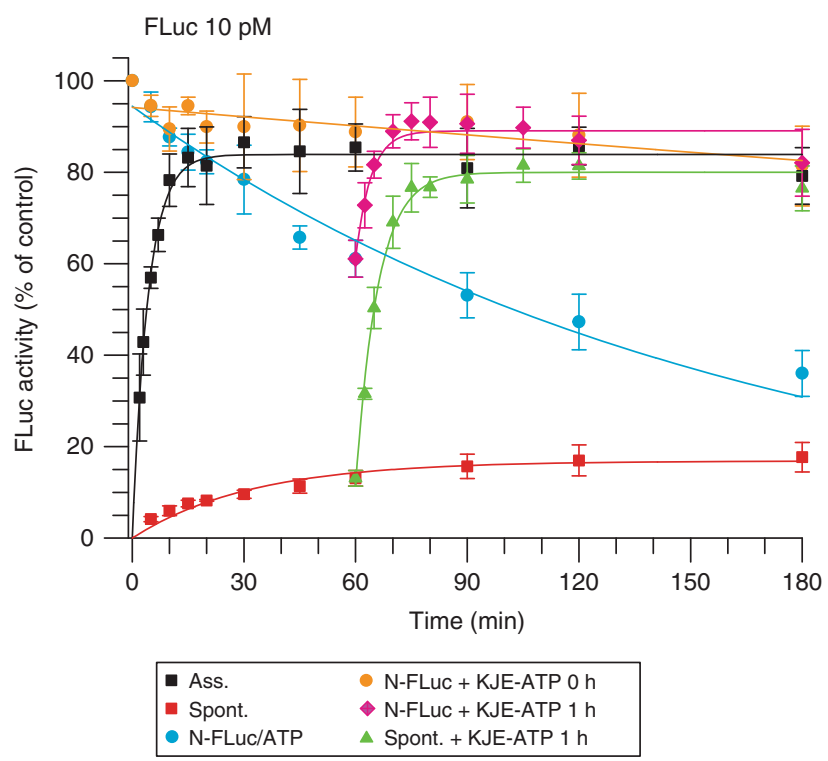

b

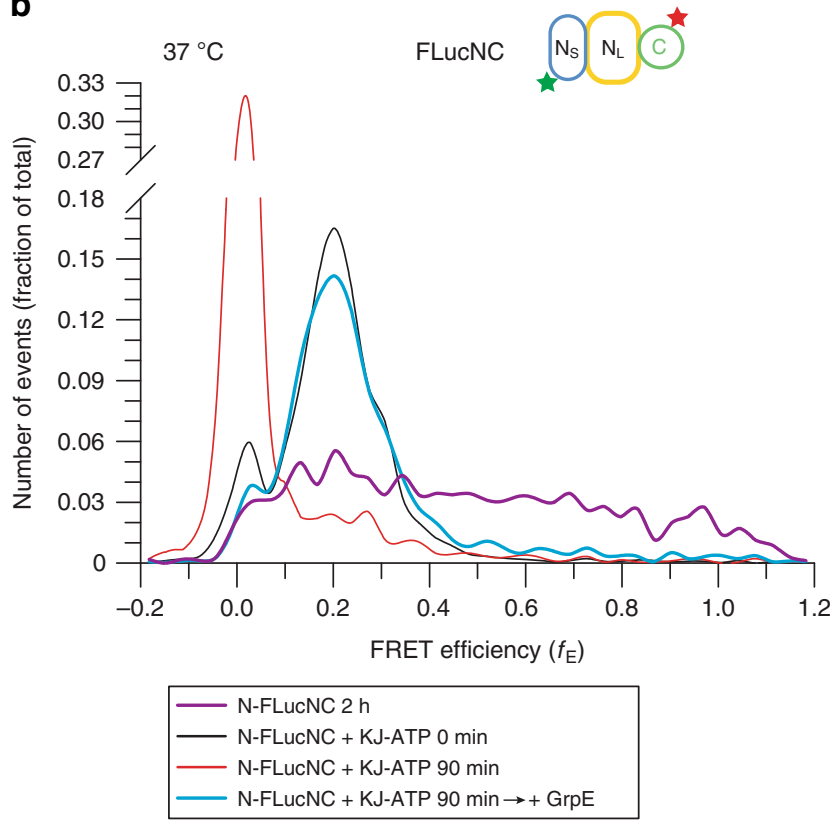

Fig. $8 \mathrm{KJE}$-accelerated folding maintains the native state of FLuc. a Luminescence activity of $10 \mathrm{pM}$ FLuc was monitored at $37^{\circ} \mathrm{C}$, in the presence or absence of KJE-ATP. Time-dependent loss of FLuc activity was monitored by shifting N-FLuc from $25^{\circ} \mathrm{C}$ to $37^{\circ} \mathrm{C}$ (blue circles). $1 \mu \mathrm{M} \mathrm{DnaK}, 0.33 \mu \mathrm{M}$ DnaJ and $1.5 \mu \mathrm{M}$ GrpE were included during temperature upshift of N-FLuc (orange circles), or added after $1 \mathrm{~h}$ incubation of $\mathrm{N}$-FLuc at $37{ }^{\circ} \mathrm{C}$ (magenta diamonds), or after $1 \mathrm{~h}$ of spontaneous refolding from denaturant at $37^{\circ} \mathrm{C}$ (green triangles). Assisted (Ass., black squares) and spontaneous (Spont., red squares) refolding are also shown. Error bars represent s.d. $(n=5)$. Ass., KJE-ATP-assisted; Spont., spontaneous folding. b N-FLucNC (10 pM) in folding buffer with PBT and ATP was shifted from $25^{\circ} \mathrm{C}$ to $37^{\circ} \mathrm{C}$ in the presence or absence of KJ. spFRET was recorded for 30 min after $2 \mathrm{~h}$ without chaperones. Upon heating in the presence of KJ-ATP, spFRET was recorded either immediately ( $0 \mathrm{~min}$ ) or after $90 \mathrm{~min}$. GrpE was added after $2 \mathrm{~h}$ to initiate refolding of FLuc from the DnaK-bound state. Representative measurements of three independent repeats are shown. Source data are provided as a Source Data file. 


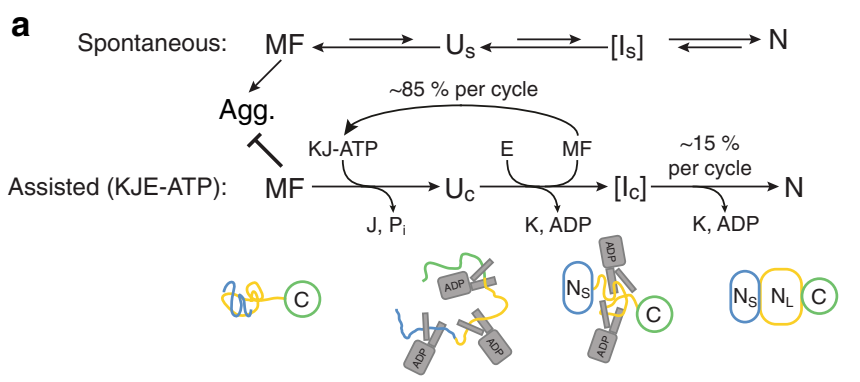

b

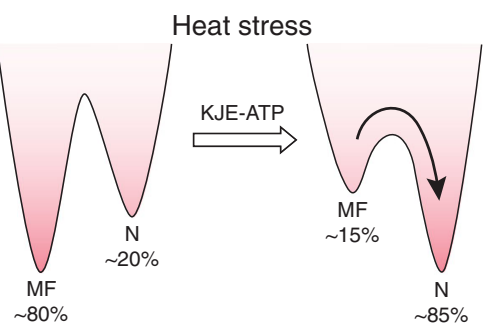

Fig. 9 Model of spontaneous and KJE-ATP assisted folding of FLuc. a MF, misfolded compact intermediate; Agg., aggregated protein; $U_{S}$, unfolded state in the absence of chaperones; $I_{S}$, folding intermediate of spontaneous folding; $U_{C}$, unfolded state in complex with DnaK; $I_{C}$, intermediate committed to fast folding; $N$, native state. Lengths of arrows reflects approximate relative rates. $\mathbf{b}$ Simplified energy diagrams to illustrate the effect of KJE-ATP in stabilizing the native state of FLuc at elevated temperature by accelerating refolding of MF.

spontaneous folding in the absence of aggregation, FLuc populates kinetically trapped, misfolded states (MF). These intermediates are aggregation-prone but remain monomeric at very low protein concentration. They are characterized by non-native interactions between the $\mathrm{N}_{S}$ and $\mathrm{N}_{\mathrm{L}}$ subdomains of FLuc, which need to be resolved to allow $\mathrm{N}$-domain folding (Fig. 9a). KJE-ATP markedly ( 20-fold) accelerates folding by two complementary activities. First, DnaK and DnaJ cooperate in an ATP hydrolysis-dependent process to unfold misfolded FLuc, thereby catalyzing escape from kinetic traps. Binding of multiple DnaK molecules converts MF to an expanded state $\left(\mathrm{U}_{\mathrm{C}}\right)$ (Fig. 9a). Similar expanded conformations for other Hsp70-bound proteins have been suggested to arise from steric repulsion between multiple DnaK molecules bound to the same substrate protein ${ }^{41,45-47}$. Second, GrpE-mediated release of DnaK directs the bound FLuc molecules towards an intermediate conformation, $\mathrm{I}_{\mathrm{C}}$, that is committed to fold fast to the native state (Fig. 9a), effectively lowering the energy barrier towards N (Fig. 9b). As shown by the single cycle experiments, this reaction is critical for acceleration of FLuc folding but is of limited efficiency $(\sim 15 \%$ of molecules). The remainder of FLuc reverts to MF states after release from DnaK, followed by unfolding upon rebinding to DnaK.

At an efficiency of $\sim 15 \%$ for fast folding per chaperone cycle, $\sim 4$ cycles are required for half-maximal folding. Each cycle would nominally consume $\sim 10$ molecules of ATP, assuming that $\sim 10$ DnaK bind to FLuc. However, FLuc molecules may not necessarily return to the fully expanded, DnaK-bound state during uninterrupted cycling and fewer DnaK may be sufficient to destabilize MF. The actual ATP consumption of the folding reaction remains to be determined.

Prior work provided evidence that DnaK can use chemical energy from ATP hydrolysis to drive substrate folding out of equilibrium $^{23,48}$. Our data are consistent with this concept, and demonstrate that in case of FLuc, stabilization of the native state can be explained by kinetic folding assistance, whereby KJE-ATP selectively accelerates the re-folding of kinetically trapped, misfolded protein (Fig. 9). In the absence of chaperone, these kinetically trapped states function effectively as a sink, especially under conditions that destabilize the native protein, such as elevated temperature.

Our observation that misfolding is avoided by a fraction of FLuc in each chaperone cycle would appear inconsistent with a mechanism of simultaneous release of the multiple bound DnaK molecules. Instead, and consistent with the H/DX-MS data, we hypothesize that KJE biases the folding pathway by a mechanism of asynchronous release of DnaK molecules. Additional support for this idea comes from NMR experiments on DnaK-bound hTRF1, a 53-residue model client ${ }^{49,50}$. hTRF1 can bind between one and three DnaK, which is suggested to result in conformational heterogeneity in the bound substrate at the onset of folding. A mechanism of asynchronous release might allow structure formation in the $\mathrm{N}_{\mathrm{S}}$-subdomain of FLuc without interference from the $\mathrm{N}_{\mathrm{L}}$-subdomain, thereby bypassing the kinetic folding trap for a fraction of molecules in each cycle (Fig. 9a). The type of inter-domain misfolding rescued by the KJE system is common in large multi-domain proteins ${ }^{36,51-53}$ and artificial polyproteins $21,35,54,55$, and might occur during de novo folding or upon heat stress-induced denaturation. Indeed, the KJE system has recently been shown to stabilize numerous multi-domain proteins against thermal denaturation in vivo (Fig. 9b) ${ }^{18}$. Our observations for the heterologous substrate FLuc may therefore more generally explain the stress-protective function of Hsp70.

The ability to accelerate protein folding by an ATP-dependent mechanism has also been demonstrated for the cylindrical GroEL/ES chaperonin ${ }^{56}$. Several proteins utilizing this system have been shown to populate dynamic folding intermediates that convert only slowly to the native state due to a long search time for the formation of native contacts ${ }^{57-59}$. Their encapsulation in the folding cage formed by GroEL and GroES results in a rate enhancement of folding by one to two orders of magnitude $57,58,60,61$, which has been attributed to an effect of entropic confinement ${ }^{56-58}$. In contrast, KJE-mediated binding and release cycles fail to accelerate the folding of these proteins but rather slow their folding kinetics ${ }^{19,58,60}$. Emerging from these findings is the concept that KJE and GroEL/ES, as major chaperone systems in the bacterial cytosol, attend to protein subsets that populate different types of kinetically trapped folding intermediates. While GroEL/ES catalyses folding of entropically stable intermediates, Hsp70 rescues misfolded states that are stabilized by non-native interactions that need to be resolved in order to allow folding.

\section{Methods}

Cloning, plasmids and strains. Full-length FLuc from Photinus pyralis was cloned into a pET3a plasmid (Supplementary Table 2). Point mutations for site-specific fluorophore labelling were introduced using the QuikChange Multi Site-Directed Mutagenesis Kit (Agilent Technologies). FLuc190 was generated by insertion of a stop codon using Q5 Site-Directed Mutagenesis Kit (New England BioLabs). GrpE was expressed from plasmid pET3a, and DnaK and DnaJ from pET11d. Plasmids, proteins and strains used or generated in this study are listed in Supplementary Table 3.

Expression and purification of FLuc and variants. Wild-type FLuc was purchased from Promega, or purified as follows. Wild-type and mutant FLuc constructs were transformed into E. coli BL21 (DE3) cells. Cells were grown at $37^{\circ} \mathrm{C}$ until $\mathrm{OD}_{600}$ reached $\sim 0.4$. The temperature was then shifted to $18^{\circ} \mathrm{C}$ and cultures were incubated for $1 \mathrm{~h}$ before addition of IPTG to a final concentration of $0.25 \mathrm{mM}$. Proteins were expressed for $16 \mathrm{~h}$ at $18^{\circ} \mathrm{C}$. Cells were harvested by centrifugation at $5000 \mathrm{~g}$ for $30 \mathrm{~min}$ and pellets were resuspended in lysis buffer $\left(50 \mathrm{mM} \mathrm{NaH} \mathrm{PO}_{4}\right.$ $\mathrm{pH}$ 8.0, $300 \mathrm{mM} \mathrm{NaCl}, 10 \mathrm{mM}$ Imidazole, $1 \mathrm{mg} \mathrm{mL}^{-1}$ lysozyme, PMSF, aprotinin, PepA, leupeptin, DNase I and Complete ${ }^{\mathrm{TM}}$, EDTA free protease inhibitor cocktail (Roche)). Cells were lysed by sonication, and the lysate was cleared by centrifugation at $66,000 \mathrm{~g}$ for $30 \mathrm{~min}$. The supernatant was loaded onto a $5 \mathrm{~mL} \mathrm{Ni-NTA}$ column (HisTrap ${ }^{\mathrm{TM}} \mathrm{HP}$, GE Healthcare Life Sciences) pre-equilibrated with equilibration buffer ( $50 \mathrm{mM} \mathrm{NaH} \mathrm{PO}_{4} \mathrm{pH} 8.0,300 \mathrm{mM} \mathrm{NaCl}, 20 \mathrm{mM}$ imidazole). Protein was eluted in $2 \mathrm{~mL}$ fractions using equilibration buffer containing $500 \mathrm{mM}$ imidazole, and purity of the fractions was assessed by $12 \%$ SDS-PAGE. Fractions 
containing pure FLuc were pooled, concentrated, and buffer exchanged into FLuc storage buffer ( $25 \mathrm{mM}$ Tris-acetate $\mathrm{pH}$ 7.8, $1 \mathrm{mM}$ EDTA, $0.2 \mathrm{M}$ ammonium sulfate, $15 \%$ glycerol, $30 \%$ ethylene glycol, $2 \mathrm{mM}$ TCEP) before snap-freezing in liquid $\mathrm{N}_{2}$ and storage at $-80^{\circ} \mathrm{C}$.

Expression and purification of chaperones. The DnaK construct was transformed into E. coli BL21 (DE3) cells. Cells were grown at $37^{\circ} \mathrm{C}$ until $\mathrm{OD}_{600}$ reached $0.6-0.8$, then expression was induced with $0.5 \mathrm{mM}$ IPTG at $30^{\circ} \mathrm{C}$. After $4-5 \mathrm{~h}$ expression, cells were harvested, resuspended in buffer $(20 \mathrm{mM}$ Tris- $\mathrm{HCl}$ pH 7.4, 1 mM EDTA, $1 \mathrm{mg} \mathrm{mL}^{-1}$ lysozyme, PMSF, aprotinin, PepA, leupeptin, DNase I and Complete ${ }^{\mathrm{TM}}$, EDTA free protease inhibitor cocktail (Roche)) and lysed as described above for FLuc by centrifugation at $5000 \mathrm{~g}$ for $30 \mathrm{~min}$. Cells were resuspended in buffer A (20 mM Tris-HCl, pH 7.4, 1 mM EDTA). The lysate was clarified as described above, and the supernatant was loaded onto a Source 30Q (GE

Healthcare, $50 \mathrm{~mL}$ ) column pre-equilibrated with buffer A. The column was washed with buffer A, and protein was eluted using buffer A/1 M NaCl. Fractions containing DnaK were pooled and buffer exchanged into HMK buffer $(20 \mathrm{mM}$ Hepes/KOH, pH 7.4, $5 \mathrm{mM} \mathrm{Mg}\left(\mathrm{OAc}_{2}\right)$ ) using a HiPrep 26/10 desalting column (GE Healthcare, $56 \mathrm{~mL}$ ) column. The sample was then loaded onto a Hiprep Heparin Sepharose FastFlow FF16/10 column (GE Healthcare) pre-equilibrated with HMK buffer. Elution was performed with a $0-500 \mathrm{mM} \mathrm{NaCl}$ gradient. Fractions containing DnaK were pooled and buffer exchanged into HMK buffer, then loaded onto a MonoQ 10/100 column (GE Healthcare). Protein was eluted with a $0-500 \mathrm{mM} \mathrm{NaCl}$ gradient, and pooled fractions were buffer exchanged into HMK buffer $/ 100 \mathrm{mM} \mathrm{NaCl}$. The eluted protein was further purified by gel filtration using a Superdex 200 16/60 column equilibrated with HMK buffer/100 mM NaCl. Fractions containing pure DnaK were pooled and concentrated before snapfreezing in liquid $\mathrm{N}_{2}$ and storage at $-80^{\circ} \mathrm{C}$.

The GrpE construct was transformed into E. coli BL21 (DE3) cells. Expression was performed as described above for DnaK. Harvested cells were resuspended in buffer $(50 \mathrm{mM}$ Tris- $\mathrm{HCl} \mathrm{pH} 8.0,10 \%$ sucrose $(\mathrm{w} / \mathrm{v}))$ and lysed with lysozyme solution containing $46 \mu \mathrm{g} \mathrm{mL}^{-1}$ spermidine, $50 \mathrm{mM}$ DTT, $50 \mathrm{mM}$ EDTA and $0.2 \mathrm{~g}$ $\mathrm{mL}^{-1}$ ammonium sulfate. For lysis, the cell suspension was incubated for $45 \mathrm{~min}$ on ice, then heated to $37^{\circ} \mathrm{C}$ for $5 \mathrm{~min}$ with gentle shaking, followed by cooling on ice for $5 \mathrm{~min}$. The lysate was clarified by centrifugation at $66,000 \mathrm{~g}$ for $30 \mathrm{~min}$ at $4{ }^{\circ} \mathrm{C}$. Ammonium sulfate was then added to a final concentration of $0.35 \mathrm{~g} \mathrm{~L}^{-1}$ and the solution was stirred for $20 \mathrm{~min}$. Following centrifugation $(66,000 \mathrm{~g}, 30 \mathrm{~min})$, the pellet was resuspended in buffer B (25 mM Hepes-KOH pH 8.0, 1 mM EDTA, 10 $\mathrm{mM} 2$-mercaptoethanol, 20\% (v/v) glycerol) and loaded onto an Enrich Q column (BioRad) pre-equilibrated with buffer B. The protein was eluted with buffer C (25 mM Hepes-KOH pH 8, 1 mM EDTA, $10 \mathrm{mM}$ 2-mercaptoethanol, 10\% (v/v) glycerol, $2 \mathrm{M} \mathrm{KCl}$ ) and then buffer exchanged into buffer B before performing a second round of ion exchange chromatography using a MonoQ column (GE Healthcare). The eluted protein was further purified by gel filtration using a HiPrep16/60 Sephacryl-S100 column (GE Healthcare) pre-equilibrated with buffer B. Fractions containing pure GrpE were pooled and concentrated before snapfreezing in liquid $\mathrm{N}_{2}$ and storage at $-80^{\circ} \mathrm{C}$.

DnaJ was expressed and purified as described previously ${ }^{6}$.

Luminescence assay of FLuc folding. Denatured FLuc was prepared by incubation of native FLuc (N-FLuc) in $5 \mathrm{M} \mathrm{GuHCl}$ and $10 \mathrm{mM}$ DTT for $1 \mathrm{~h}$ at $25^{\circ} \mathrm{C}$. Spontaneous folding was initiated by 100 -fold dilution into folding buffer $(25 \mathrm{mM}$ Hepes-KOH pH 7.5, $100 \mathrm{mM} \mathrm{KCl,} 10 \mathrm{mM} \mathrm{Mg}(\mathrm{OAc})_{2}, 2 \mathrm{mM}$ DTT, 0.05\% T20), resulting in a final $\mathrm{GuHCl}$ concentration of $50 \mathrm{mM}$. Assisted folding reactions were initiated by 100 -fold dilution into folding buffer containing DnaK, DnaJ, GrpE and $5 \mathrm{mM}$ ATP. Chaperone concentrations were optimized to maximize refolding yield at the concentration of FLuc used and avoid inhibitory effects of excess chaperones $^{29}$. Reactions with $100 \mathrm{nM}$ FLuc contained $3 \mu \mathrm{M}$ DnaK, $1 \mu \mathrm{M}$ DnaJ and $1.5 \mu \mathrm{M}$ GrpE. Reactions with $1 \mathrm{nM}, 0.1 \mathrm{nM}$ or $50 \mathrm{pM}$ FLuc contained $0.3 \mu \mathrm{M}$ DnaK, 0.1 $\mu \mathrm{M}$ DnaJ and $0.5 \mu \mathrm{M}$ GrpE. Reactions with $10 \mathrm{pM}$ FLuc (at $37^{\circ} \mathrm{C}$ ) contained $1 \mu \mathrm{M}$ DnaK, $0.33 \mu \mathrm{M}$ DnaJ and $1.5 \mu \mathrm{M}$ GrpE. Reactions were performed at $25^{\circ} \mathrm{C}$ unless otherwise indicated.

Luminescence was measured using a Lumat LB9508 luminometer (Berthold Technologies). At indicated time points, aliquots of folding reactions or N-FLuc were diluted at least 25-fold in Luciferase Assay System solution (Promega) and luminescence was measured for $2 \mathrm{~s}$ at $25^{\circ} \mathrm{C}$. Folding yields were calculated by normalizing to the luminescence activity of N-FLuc. Prior to analysis, N-FLuc stocks were centrifuged at $20,000 \mathrm{~g}$ for $30 \mathrm{~min}$ to remove aggregates. All measurements were performed at least three times. Folding curves were fit using Sigmaplot 14.0 (Systat Software).

Rescue of FLuc activity was performed by adding $0.3 \mu \mathrm{M}$ DnaK, $0.1 \mu \mathrm{M}$ DnaJ, $0.5 \mu \mathrm{M}$ GrpE and $5 \mathrm{mM} \mathrm{ATP}$ at $2 \mathrm{~h}, 4 \mathrm{~h}$ or $8 \mathrm{~h}$ after initiation of spontaneous folding as described above.

To analyse FLuc folding in a single round of chaperone action, GuHCldenatured FLuc was diluted to $100 \mathrm{pM}$ in folding buffer containing $0.3 \mu \mathrm{M}$ DnaK, $0.1 \mu \mathrm{M}$ DnaJ, $5 \mathrm{mM}$ ATP and $125 \mu \mathrm{M}$ peptide NR peptide (GSGNRLLLTG). After incubation for $1 \mathrm{~min}$, luminescence activity was measured at intervals as described above. As controls, peptide NR was added to the chaperone mixture prior to addition of FLuc, or after 5 min of chaperone-assisted folding.
Reactions at $37^{\circ} \mathrm{C}$ were performed as described above, except that folding was assayed at $10 \mathrm{pM}$ FLuc, and assisted folding reactions contained $1 \mu \mathrm{M}$ DnaK, $0.33 \mu \mathrm{M}$ DnaJ and $1.5 \mu \mathrm{M}$ GrpE.

Fluorophore labelling of proteins. For spFRET experiments, double-cysteine mutant variants of FLuc were labelled with Atto532-maleimide and Atto647Nmaleimide (ATTO-TEC) at a 1.5-fold molar excess of each fluorophore. Before labelling, FLuc cysteine residues were reduced by incubation with $2 \mathrm{mM}$ tris(2carboxyethyl)phosphine (TCEP), which was subsequently removed using a Nap5 column (GE Healthcare). Labelling was performed in labelling buffer $(25 \mathrm{mM}$ Hepes-KOH pH 7.5, $100 \mathrm{mM} \mathrm{KCl,} 10 \mathrm{mM} \mathrm{Mg(OAc)} 2,5 \mathrm{mM}$ ATP, $100 \mu \mathrm{M}$ phenylbenzothiazole (PBT)) for $2 \mathrm{~h}$ on ice. After labelling, free dye was removed using a Nap5 column pre-equilibrated with FLuc storage buffer. Labelling efficiencies were typically $\sim 70 \%$, and the absence of free dye was confirmed using FCS. The specificity of labelling was assessed by comparing the measured FRET efficiency distribution with the distance between labelling residues according to the crystal structure of FLuc (PDB: 1LCI ${ }^{26}$

For FCS and FCCS experiments, wild-type FLuc was labelled at the N-terminus with either Alexa532 or Alexa647 N-hydroxysuccinimide (NHS) ester (Molecular Probes) at a 5-fold molar excess. Labelling was performed at $4{ }^{\circ} \mathrm{C}$ for $90 \mathrm{~min}$ in 0.1 $\mathrm{M} \mathrm{NaHCO}_{3} \mathrm{pH} 8.3$, followed by removal of free dye using a Nap5 column preequilibrated with FLuc storage buffer. Labelling efficiencies were typically $\sim 75 \%$. Double-labelled FLuc for FCCS experiments was prepared by incubation of FLucNC (D19C/S504C) with Alexa532- and Alexa647-maleimide. Labelling was performed as described above for the spFRET constructs.

For FCS experiments, FLucNC was labelled with Atto647N-maleimide as described above for spFRET constructs.

For analytical ultracentrifugation experiments, FLucNC was labelled with a 6fold excess of Atto532-maleimide. Labelling was performed in labelling buffer containing $4 \mathrm{M} \mathrm{GuHCl}$, and without PBT, for $1 \mathrm{~h}$ at $25^{\circ} \mathrm{C}$. Free dye was removed using a Nap5 column equilibrated with labelling buffer containing $4 \mathrm{M} \mathrm{GuHCl}$.

DnaJ was labelled at the $\mathrm{N}$-terminus with Alexa647 N-hydroxysuccinimide ester (Molecular Probes) at a 2 -fold molar excess. Labelling was performed at $4{ }^{\circ} \mathrm{C}$

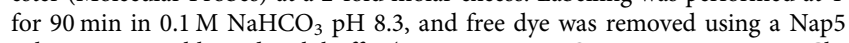
column pre-equilibrated with buffer ( $25 \mathrm{mM}$ Hepes-KOH pH 7.5, $100 \mathrm{mM} \mathrm{KCl,} 10$ $\mathrm{mM} \mathrm{Mg}(\mathrm{OAc})_{2}, 2 \mathrm{mM} \mathrm{DTT}, 10 \%$ (v/v) glycerol). Labelling efficiencies were typically $\sim 80 \%$.

Fluorescence correlation and cross-correlation spectroscopy. FCS and dcFCCS measurements using pulsed interleaved excitation (PIE) ${ }^{33}$ were performed on a MicroTime 200 inverse time-resolved fluorescence microscope (PicoQuant) as previously described ${ }^{62}$. Experiments were performed at a constant temperature of $20^{\circ} \mathrm{C}$ unless otherwise stated. Pulsed diode lasers at $530 \mathrm{~nm}$ (LDH-P-FA-530) and $640 \mathrm{~nm}$ (LDH-PC-640B) were used for excitation of Alexa532 and Alexa 647, respectively. For FCS measurements, the laser power was set to $60 \mu \mathrm{W}$ measured before the major dichroic filter. For FCCS measurements, each laser was set to 40 $\mu \mathrm{W}$. The lasers were pulsed with a rate of $26.7 \mathrm{mHz}$. Measurements were performed using a water immersion objective (×60 1.2 NA, Olympus) in the sample cuvette (Ibidi). The emitted fluorescence was separated from excitation light by a dichroic mirror (Z532/635RPC), guided through a pinhole $(75 \mu \mathrm{m})$ and in case of cross-correlation split according to wavelength by a beam splitter (600 DCXR) onto photon avalanche diodes (SPADs) (PDM series, MPD). The emission light was filtered by emission bandpass filters (HQ 690/70 and HQ 580/70, Chromas) in front of the respective detector. Detection was performed using time correlated single photon counting, making it possible to correlate any given photon with the excitation source. For FCS measurements, after-pulsing artifacts were removed by fluorescence lifetime filters (SymPhoTime, PicoQuant) ${ }^{63}$. Correlation plots were fitted with triplet diffusion equation using SymPhoTime 32 (PicoQuant). The confocal volume $\left(\mathrm{V}_{\text {eff }}\right)$ was calibrated daily using Atto655-maleimide dye.

The diffusion time of 50 pM FLucNC-Atto532 was measured by FCS for FLuc in the native state or in complex with DnaK, prepared as described above for the folding assays. Autocorrelation was recorded for $30 \mathrm{~min}$.

FCCS measurements were performed to measure intermolecular association during spontaneous folding of FLuc. FLuc-Alexa532 and FLuc-Alexa647 were denatured as described above, then diluted to different final concentrations in folding buffer, maintaining a 1:1 molar ratio of labelled proteins. Where indicated, reactions were supplemented with different concentrations of denatured, un-labelled FLuc. FCCS was recorded for $30 \mathrm{~min}$ at $25^{\circ} \mathrm{C}$, or $1 \mathrm{~h}$ at $37^{\circ} \mathrm{C}$. Reactions were held at $25^{\circ} \mathrm{C}$ or $37^{\circ} \mathrm{C}$, as indicated, using a temperature controlled chamber (Ibidi Heating System). Double-labelled FLucNC ( $5 \mathrm{nM})$ was used as a positive control.

To determine the composition of the chaperone-FLuc complex, complexes were prepared as described above, using pairs of labelled components, and FCCS was recorded for $30 \mathrm{~min}$. Complexes with labelled DnaJ were prepared with $0.3 \mu \mathrm{M}$ DnaK, 10 nM DnaJ-Alexa647, 90 nM un-labelled DnaJ and 10 nM FLuc-Alexa532. To test whether complexes contained multiple FLuc molecules, complexes were prepared with $0.3 \mu \mathrm{M}$ DnaK, $0.1 \mu \mathrm{M}$ DnaJ, 50 pM FLuc-Alexa532 and 50 pM FLucAlexa647. Double-labelled FLucNC-Alexa532/647 at $50 \mathrm{pM}$ was used as a positive control. 
Single-pair fluorescence resonance energy transfer (spFRET). spFRET experiments were performed on a MicroTime 200 inverse time-resolved fluorescence microscope (PicoQuant) using pulsed interleaved excitation as described above. Temperature was maintained at $20^{\circ} \mathrm{C}$ unless otherwise stated. Each laser was set to $40 \mu \mathrm{W}$, measured before the major dichroic filter. Unless otherwise stated, all spFRET experiments were performed at $50 \mathrm{pM}$ FLuc to ensure that the probability of having more than one particle in the confocal volume is $<1 \%$. FRET was recorded for $15 \mathrm{~min}$ (chaperone-assisted folding at $20^{\circ} \mathrm{C}$ ), $30 \mathrm{~min}$ (chaperoneassisted folding at $37^{\circ} \mathrm{C}$ ) or $1 \mathrm{~h}$ (spontaneous folding). Assisted folding reactions contained $0.3 \mu \mathrm{M}$ DnaK, $0.1 \mu \mathrm{M}$ DnaJ and $0.5 \mu \mathrm{M}$ GrpE where indicated. All experiments were performed in folding buffer containing $5 \mathrm{mM}$ ATP and $50 \mu \mathrm{M}$ PBT and performed at least three times (residual GuHCl $50 \mathrm{mM}$ ).

Data were analysed using a burst intensity approach ${ }^{64}$ in SymPhoTime (PicoQuant). A single molecule diffusing through the confocal observation volume results in a burst in fluorescence intensity. A burst was considered a significant event when it contained more than 25 photons in a $1 \mathrm{~ms}$ bin time window. In addition, a threshold of 15 photons following red excitation was used to confirm the presence of a functional acceptor fluorophore. To constitute a significant measurement, at least 500 events were analysed for a single spFRET distribution histogram. FRET efficiencies were calculated from fluorescence intensities of donor $\left(I_{\mathrm{D}}\right)$ and acceptor $\left(I_{\mathrm{A}}\right)$ fluorophore by the equation:

$$
E=\frac{I_{\mathrm{A}}}{I_{\mathrm{A}}+\gamma I_{\mathrm{D}}},
$$

where $\gamma=\left(\Phi_{\mathrm{A}} \eta_{\mathrm{A}} / \Phi_{\mathrm{D}} \eta_{\mathrm{D}}\right)$ denotes a correction factor for differences in quantum yields $(\Phi)$ and detection efficiencies $(\eta)^{33}$ and has been found to be 0.9 for the FRET pair used ${ }^{62}$. Average intensity values of spectral crosstalk and direct excitation of acceptor fluorophores by the green laser were subtracted.

The resulting FRET efficiency histograms were fit to a sum of Gaussian distributions using Origin (OriginLabs). To quantify the fraction of native molecules during spontaneous folding, the area of the histogram corresponding to N-FLuc was divided by the total area of the histogram at each folding time point $(0-10 \mathrm{~h})$. This fraction was plotted against refolding time and then fitted to a single exponential function, yielding approximate rates of refolding. Data were recorded continuously over the $10 \mathrm{~h}$ period, then subdivided for analysis using a sliding window. The duration of the window was $30 \mathrm{~min}$ for early time points, and $1 \mathrm{~h}$ for later time points. Since folding continues during the measurement, time points are given as the midpoint of each recording interval, for example, the earliest time point $(15 \mathrm{~min})$ corresponds to data collected in the $0-30 \mathrm{~min}$ window. For FLucC, data for the first time point were summed over $1 \mathrm{~h}$ in order to include sufficient FRET events to generate a histogram that could be reliably fitted. The first time point for the spontaneous folding of FLucC is therefore marked as $30 \mathrm{~min}$.

spFRET measurements at $37^{\circ} \mathrm{C}$ were performed as described above, and the temperature was controlled using an Ibidi Heating System.

Analytical ultracentrifugation (AUC). To avoid aggregation, $200 \mathrm{nM}$ FLucAtto532 (prepared as described above) was analysed in folding buffer with $5 \mathrm{M}$ $\mathrm{GuHCl}$. The DnaK-FLuc complex was prepared by dilution of GuHCl-denatured FLuc-Atto532 to $200 \mathrm{nM}$ in folding buffer containing $3 \mu \mathrm{M}$ DnaK, $1 \mu \mathrm{M}$ DnaJ and $5 \mathrm{mM}$ ATP. DnaK-FLuc complexes prepared under these conditions produced folded FLuc with a similar rate and yield to control reactions using un-labelled protein. Three independent replicates were performed for each condition.

Sedimentation velocity experiments were performed on an Optima XL-I analytical centrifuge (Beckman Inc., Indianapolis, In, U.S.A.) using an An-60 Ti rotor and double-sector $12 \mathrm{~mm}$ centrepieces. Buffer density was measured as $1.02054 \mathrm{~kg} \mathrm{~L}^{-1}$ using a DMA 5000 densitometer (Anton Paar, Graz, Austria). Protein concentration distribution was monitored at $532 \mathrm{~nm}$, using 184,000 xg rotor speed. Time-derivative analysis was computed using the SEDFIT software package ${ }^{65}$, resulting in a $c(s)$ distribution and an estimate for the molecular weight (from sedimentation coefficient and the diffusion coefficient, inferred from the peak width)

Hydrogen/deuterium exchange-mass spectrometry (H/DX-MS). For equilibrium H/DX-MS, native and DnaK-bound FLuc were prepared essentially as described above for the luminescence folding assays. N-FLuc was prepared at final concentration of $1 \mu \mathrm{M}$ in HDX buffer (folding buffer with $5 \mathrm{mM}$ ATP, and without $0.05 \% \mathrm{~T} 20$ ) and the DnaK-FLuc complex was prepared by dilution of $\mathrm{GuHCl}-$ denatured FLuc to final $1 \mu \mathrm{M}$ in HDX buffer containing $10 \mu \mathrm{M}$ DnaK and $3.3 \mu \mathrm{M}$ DnaJ (residual GuHCl $50 \mathrm{mM}$ ). DnaK-FLuc complexes prepared under these conditions produced folded FLuc with a similar rate and yield to control reactions at lower concentrations of FLuc and chaperones described above. Three independent replicates were performed for each condition.

Deuterium exchange was initiated by 10 -fold dilution into deuteration buffer (HDX buffer prepared in $\mathrm{D}_{2} \mathrm{O}$ ), followed by incubation at $25^{\circ} \mathrm{C}$ for $10 \mathrm{~s}, 30 \mathrm{~s}, 100 \mathrm{~s}$ or $300 \mathrm{~s}$. DnaK-FLuc complexes were analysed immediately after they were prepared, to avoid release of FLuc over prolonged incubation times. Exchange reactions were quenched by addition of an equal volume of ice-cold quench buffer (100 mM sodium phosphate $\mathrm{pH}$ 2.1, $10 \mathrm{mM}$ tris(2-carboxyethyl)phosphine (TCEP), $1 \mathrm{M} \mathrm{GuHCl}$ ), to a final $\mathrm{pH}$ of 2.6 .
For pulsed-label H/DX, DnaK-FLuc complexes were prepared as described above for the equilibrium H/DX experiments, and FLuc folding was initiated by addition of $5 \mu \mathrm{M} \mathrm{GrpE}$. At different folding times ( $10 \mathrm{~s}, 5 \mathrm{~min}, 30 \mathrm{~min}$ ), $5 \mu \mathrm{l}$ aliquots of the reaction were withdrawn and added to $45 \mu \mathrm{l}$ deuteration buffer. Deuterium labelling was allowed to proceed for $10 \mathrm{~s}$, before quenching by addition of $50 \mu \mathrm{l}$ quench buffer containing $20 \%$ glycerol, and snap-freezing in liquid $\mathrm{N}_{2}$. Samples were stored at $-80^{\circ} \mathrm{C}$ for no longer than one week before analysis.

Proteolysis, chromatography, peptide-mass analysis and data processing were performed as described previously ${ }^{66}$. To process spectra with bimodal patterns, all isotopes of the entire bimodal distribution were selected for processing, and relative deuterium incorporation was calculated based on the centroid of the isotopic distribution. Experiments were performed at least three times, and the error of mass measurement was $<0.2 \mathrm{Da}$. All experiments were performed under identical conditions. Deuterium levels were therefore not corrected for backexchange and all data are reported as relative. Sequence coverage was $\sim 95 \%$ for N-FLuc, $\sim 60 \%$ for FLuc in complex with DnaK, and $\sim 60 \%$ for FLuc in the pulsed-label experiment.

The mass spectrometry proteomics data have been deposited to the ProteomeXchange Consortium via the PRIDE ${ }^{67}$ partner repository with the dataset identifier PXD016509.

Computational prediction of DnaK binding sites on FLuc. DnaK binding sites on FLuc were predicted using the LIMBO web server ${ }^{37}$. Heptapeptide motifs with a score $>10$ were considered to be high confidence DnaK binding sites. The sequence of Photinus pyralis luciferase (UniprotKB P08659) was used as an input.

Reporting summary. Further information on research design is available in the Nature Research Reporting Summary linked to this article.

\section{Data availability}

All data presented in this study are available within the figures and in the Supplementary Information, including the Source Data file linked to this article. The source data underlying Fig. 2b, c, 3b-k, 4a-e, 6b, 7 and 8a, b and Supplementary Figs. 1a-e, 2a-h, 3a, b, $4 a-c, 5 a, b, 6,8 a-c, 9 a, b$ and 10 are provided as a Source Data file. The search and raw files for the H/DX data are available via ProteomeXchange (PRIDE) with identifier PXD016509, and other data are available from the corresponding authors upon reasonable request.

Received: 19 September 2019; Accepted: 17 December 2019; Published online: 17 January 2020

\section{References}

1. Balchin, D., Hayer-Hartl, M. \& Hartl, F. U. In vivo aspects of protein folding and quality control. Science 353, aac4354 (2016).

2. Mayer, M. P. \& Gierasch, L. M. Recent advances in the structural and mechanistic aspects of Hsp70 molecular chaperones. J. Biol. Chem. 294, 2085-2097 (2019)

3. Rosenzweig, R., Nillegoda, N. B., Mayer, M. P. \& Bukau, B. The Hsp70 chaperone network. Nat. Rev. Mol. Cell Biol. 20, 665-680 (2019).

4. Zhu, X. et al. Structural analysis of substrate binding by the molecular chaperone DnaK. Science 272, 1606-1614 (1996).

5. Szabo, A. et al. The ATP hydrolysis-dependent reaction cycle of the Escherichia coli Hsp70 system DnaK, DnaJ, and GrpE. Proc. Natl Acad. Sci. USA 91, 10345-10349 (1994).

6. Langer, T. et al. Successive action of DnaK, DnaJ and GroEL along the pathway of chaperone-mediated protein folding. Nature 356, 683-689 (1992).

7. Kityk, R., Kopp, J., Sinning, I. \& Mayer, M. P. Structure and dynamics of the ATP-bound open conformation of Hsp70 chaperones. Mol. Cell 48, 863-874 (2012).

8. Qi, R. et al. Allosteric opening of the polypeptide-binding site when an Hsp70 binds ATP. Nat. Struct. Mol. Biol. 20, 900-907 (2013).

9. Zhuravleva, A. \& Gierasch, L. M. Substrate-binding domain conformational dynamics mediate Hsp70 allostery. Proc. Natl Acad. Sci. USA 112, E2865-E2873 (2015).

10. Kityk, R., Kopp, J. \& Mayer, M. P. Molecular mechanism of J-domaintriggered ATP hydrolysis by Hsp70 chaperones. Mol. Cell 69, 227-237.e4 (2018).

11. Mayer, M. P. Hsp70 chaperone dynamics and molecular mechanism. Trends Biochem. Sci. 38, 507-514 (2013).

12. Sikor, M., Mapa, K., von Voithenberg, L. V., Mokranjac, D. \& Lamb, D. C. Real-time observation of the conformational dynamics of mitochondrial Hsp70 by spFRET. EMBO J. 32, 1639-1649 (2013). 
13. Schroder, H., Langer, T., Hartl, F. U. \& Bukau, B. DnaK, DnaJ and GrpE form a cellular chaperone machinery capable of repairing heat-induced protein damage. EMBO J. 12, 4137-4144 (1993).

14. De Los Rios, P. \& Goloubinoff, P. Hsp70 chaperones use ATP to remodel native protein oligomers and stable aggregates by entropic pulling. Nat. Struct. Mol. Biol. 23, 766-769 (2016).

15. Teter, S. A. et al. Polypeptide flux through bacterial Hsp70: DnaK cooperates with trigger factor in chaperoning nascent chains. Cell 97, 755-765 (1999).

16. Deuerling, E., Schulze-Specking, A., Tomoyasu, T., Mogk, A. \& Bukau, B. Trigger factor and DnaK cooperate in folding of newly synthesized proteins. Nature 400, 693-696 (1999).

17. Calloni, G. et al. DnaK functions as a central hub in the E. coli chaperone network. Cell Rep. 1, 251-264 (2012).

18. Zhao, L. et al. The Hsp70 chaperone system stabilizes a thermo-sensitive subproteome in E. coli. Cell Rep. 28, 1335-1345.e1336 (2019).

19. Kerner, M. J. et al. Proteome-wide analysis of chaperonin-dependent protein folding in Escherichia coli. Cell 122, 209-220 (2005).

20. Genevaux, P. et al. In vivo analysis of the overlapping functions of DnaK and trigger factor. EMBO Rep. 5, 195-200 (2004).

21. Nunes, J. M., Mayer-Hartl, M., Hartl, F. U. \& Muller, D. J. Action of the Hsp70 chaperone system observed with single proteins. Nat. Commun. 6, 6307 (2015).

22. Sharma, S. K., De los Rios, P., Christen, P., Lustig, A. \& Goloubinoff, P. The kinetic parameters and energy cost of the Hsp70 chaperone as a polypeptide unfoldase. Nat. Chem. Biol. 6, 914-920 (2010).

23. Goloubinoff, P., Sassi, A. S., Fauvet, B., Barducci, A. \& De Los Rios, P. Chaperones convert the energy from ATP into the nonequilibrium stabilization of native proteins. Nat. Chem. Biol. 14, 388-395 (2018).

24. Frydman, J., Nimmesgern, E., Ohtsuka, K. \& Hartl, F. U. Folding of nascent polypeptide chains in a high molecular mass assembly with molecular chaperones. Nature 370, 111-117 (1994).

25. Agashe, V. R. et al. Function of trigger factor and DnaK in multidomain protein folding: increase in yield at the expense of folding speed. Cell 117, 199-209 (2004).

26. Conti, E., Franks, N. P. \& Brick, P. Crystal structure of firefly luciferase throws light on a superfamily of adenylate-forming enzymes. Structure 4, 287-298 (1996).

27. Frydman, J., Erdjument-Bromage, H., Tempst, P. \& Hartl, F. U. Cotranslational domain folding as the structural basis for the rapid de novo folding of firefly luciferase. Nat. Struct. Biol. 6, 697-705 (1999).

28. Rampelt, H. et al. Metazoan Hsp70 machines use Hsp110 to power protein disaggregation. $E M B O$ J. 31, 4221-4235 (2012).

29. Moran Luengo, T., Kityk, R., Mayer, M. P. \& Rudiger, S. G. D. Hsp90 breaks the deadlock of the Hsp70 chaperone system. Mol. Cell 70, 545-552 (2018). e549.

30. de Wet, J. R., Wood, K. V., Helinski, D. R. \& DeLuca, M. Cloning firefly luciferase. Methods Enzymol. 133, 3-14 (1986).

31. Herbst, R., Schafer, U. \& Seckler, R. Equilibrium intermediates in the reversible unfolding of firefly (Photinus pyralis) luciferase. J. Biol. Chem. 272, 7099-7105 (1997).

32. Herbst, R., Gast, K. \& Seckler, R. Folding of firefly (Photinus pyralis) luciferase: aggregation and reactivation of unfolding intermediates. Biochemistry 37, 6586-6597 (1998)

33. Muller, B. K., Zaychikov, E., Brauchle, C. \& Lamb, D. C. Pulsed interleaved excitation. Biophys. J. 89, 3508-3522 (2005).

34. Lakowicz, J. R. Principles of Fluorescence Spectroscopy. Vol. 1 (Springer, Berlin., 2006).

35. Mashaghi, A., Mashaghi, S. \& Tans, S. J. Misfolding of luciferase at the singlemolecule level. Angew. Chem. Int. Ed. Engl. Chem. 53, 10390-10393 (2014).

36. Scholl, Z. N., Yang, W. \& Marszalek, P. E. Competing pathways and multiple folding nuclei in a large multidomain protein, Luciferase. Biophys. J. 112, 1829-1840 (2017).

37. van Durme, J. et al. Accurate prediction of DnaK-peptide binding via homology modelling and experimental data. PLoS Comput. Biol. 5, e1000475 (2009).

38. Thompson, A. D., Bernard, S. M., Skiniotis, G. \& Gestwicki, J. E. Visualization and functional analysis of the oligomeric states of Escherichia coli heat shock protein 70 (Hsp70/DnaK). Cell Stress Chaperones 17, 313-327 (2012).

39. Engen, J. R. \& Smith, D. L. Investigating protein structure and dynamics by hydrogen exchange MS. Anal. Chem. 73, 256a-265a (2001).

40. Wales, T. E. \& Engen, J. R. Hydrogen exchange mass spectrometry for the analysis of protein dynamics. Mass Spectrom. Rev. 25, 158-170 (2006).

41. Sekhar, A., Rosenzweig, R., Bouvignies, G. \& Kay, L. E. Mapping the conformation of a client protein through the Hsp70 functional cycle. Proc. Natl Acad. Sci. USA 112, 10395-10400 (2015).

42. Pierpaoli, E. V., Gisler, S. M. \& Christen, P. Sequence-specific rates of interaction of target peptides with the molecular chaperones DnaK and DnaJ. Biochemistry 37, 16741-16748 (1998).
43. Mayer, M. P., Rudiger, S. \& Bukau, B. Molecular basis for interactions of the DnaK chaperone with substrates. Biol. Chem. 381, 877-885 (2000).

44. Gupta, R. et al. Firefly luciferase mutants as sensors of proteome stress. Nat. Methods 8, 879-884 (2011).

45. Kellner, R. et al. Single-molecule spectroscopy reveals chaperone-mediated expansion of substrate protein. Proc. Natl Acad. Sci. USA 111, 13355-13360 (2014).

46. Sekhar, A., Rosenzweig, R., Bouvignies, G. \& Kay, L. E. Hsp70 biases the folding pathways of client proteins. Proc. Natl Acad. Sci. USA 113 , E2794-E2801 (2016).

47. Dahiya, V. et al. Coordinated conformational processing of the tumor suppressor protein p53 by the Hsp70 and Hsp90 chaperone machineries. Mol. Cell 74, 816-830.e817 (2019).

48. $\mathrm{Xu}, \mathrm{H}$. Cochaperones enable Hsp70 to use ATP energy to stabilize native proteins out of the folding equilibrium. Sci. Rep. 8, 13213 (2018).

49. Rosenzweig, R., Sekhar, A., Nagesh, J. \& Kay, L. E. Promiscuous binding by Hsp70 results in conformational heterogeneity and fuzzy chaperone-substrate ensembles. eLife 6, https://doi.org/10.7554/eLife.28030 (2017).

50. Sekhar, A., Nagesh, J., Rosenzweig, R. \& Kay, L. E. Conformational heterogeneity in the Hsp70 chaperone-substrate ensemble identified from analysis of NMR-detected titration data. Prot. Sci. 26, 2207-2220 (2017).

51. Netzer, W. J. \& Hartl, F. U. Recombination of protein domains facilitated by co-translational folding in eukaryotes. Nature 388, 343-349 (1997).

52. Borgia, M. B. et al. Single-molecule fluorescence reveals sequence-specific misfolding in multidomain proteins. Nature 474, 662-665 (2011).

53. Pechmann, S. \& Frydman, J. Evolutionary conservation of codon optimality reveals hidden signatures of cotranslational folding. Nat. Struct. Mol. Biol. 20, 237-243 (2013).

54. Mashaghi, A. et al. Reshaping of the conformational search of a protein by the chaperone trigger factor. Nature 500, 98-101 (2013).

55. Perales-Calvo, J., Giganti, D., Stirnemann, G. \& Garcia-Manyes, S. The forcedependent mechanism of DnaK-mediated mechanical folding. Sci. Adv. 4, eaaq0243 (2018).

56. Hayer-Hartl, M., Bracher, A. \& Hartl, F. U. The GroEL-GroES chaperonin machine: A nano-cage for protein folding. Trends Biochem. Sci. 41, 62-76 (2016).

57. Chakraborty, K. et al. Chaperonin-catalyzed rescue of kinetically trapped states in protein folding. Cell 142, 112-122 (2010).

58. Georgescauld, F. et al. GroEL/ES chaperonin modulates the mechanism and accelerates the rate of TIM-barrel domain folding. Cell 157, 922-934 (2014).

59. Ye, X., Mayne, L., Kan, Z. Y. \& Englander, S. W. Folding of maltose binding protein outside of and in GroEL. Proc. Natl Acad. Sci. USA 115, 519-524 (2018).

60. Tang, Y. C. et al. Structural features of the GroEL-GroES nano-cage required for rapid folding of encapsulated protein. Cell 125, 903-914 (2006).

61. Weaver, J. et al. GroEL actively stimulates folding of the endogenous substrate protein PepQ. Nat. Commun. 8, 15934 (2017).

62. Gupta, A. J., Haldar, S., Milicic, G., Hartl, F. U. \& Hayer-Hartl, M. Active cage mechanism of chaperonin-assisted protein folding demonstrated at singlemolecule level. J. Mol. Biol. 426, 2739-2754 (2014).

63. Enderlein, J., Gregor, I., Patra, D., Dertinger, T. \& Kaupp, U. B. Performance of fluorescence correlation spectroscopy for measuring diffusion and concentration. Chemphyschem 6, 2324-2336 (2005)

64. Deniz, A. A. et al. Single-pair fluorescence resonance energy transfer on freely diffusing molecules: observation of Forster distance dependence and subpopulations. Proc. Natl Acad. Sci. USA 96, 3670-3675 (1999).

65. Schuck, P. Size-distribution analysis of macromolecules by sedimentation velocity ultracentrifugation and lamm equation modeling. Biophys. J. 78, 1606-1619 (2000).

66. Balchin, D., Milicic, G., Strauss, M., Hayer-Hartl, M. \& Hartl, F. U. Pathway of actin folding directed by the eukaryotic chaperonin TRiC. Cell 174, 1507-1521.e1516 (2018)

67. Perez-Riverol, Y. et al. The PRIDE database and related tools and resources in 2019: improving support for quantification data. Nucleic Acids Res. 47, D442-D450 (2019).

68. Hartl, F. U. \& Hayer-Hartl, M. Converging concepts of protein folding in vitro and in vivo. Nat. Struct. Mol. Biol. 16, 574-581 (2009).

\section{Acknowledgements}

The authors thank S. Gärtner, R. Lange, and N. Wischnewski for technical assistance and A. Bracher for advice and discussion. S. Uebel from the MPIB core facility is acknowledged for performing analytical ultracentrifugation. This work was supported by a grant from the Deutsche Forschungsgemeinschaft (DFG) (SFB1035) to M.H-H. and F.U.H. R.I. was supported by a DFG fellowship through the Graduate School of Quantitative Biosciences Munich (QBM). D.B. acknowledges funding by EMBO (Long-term Fellowship ALTF 1116-2014). 


\section{Author contributions}

R.I. and D.B. together with M.H-H. and F.U.H. designed the experiments presented. R.I performed all the experiments except the $\mathrm{HD} / \mathrm{X}$ measurements, which were performed by D.B. D.B., M.H.-H. and F.U.H. supervised the project and all authors participated in data interpretation. D.B., M.H.-H. and F.U.H. wrote the manuscript with contributions from R.I. F.U.H. and M.H-H. conceived the project.

\section{Competing interests}

The authors declare no competing interests.

\section{Additional information}

Supplementary information is available for this paper at https://doi.org/10.1038/s41467019-14245-4.

Correspondence and requests for materials should be addressed to D.B., M.H.-H. or F.U.H.

Peer review information Nature Communications thanks Timothy Street and the other, anonymous, reviewer(s) for their contribution to the peer review of this work.
Reprints and permission information is available at http://www.nature.com/reprints

Publisher's note Springer Nature remains neutral with regard to jurisdictional claims in published maps and institutional affiliations.

(c) (i) Open Access This article is licensed under a Creative Commons Attribution 4.0 International License, which permits use, sharing, adaptation, distribution and reproduction in any medium or format, as long as you give appropriate credit to the original author(s) and the source, provide a link to the Creative Commons license, and indicate if changes were made. The images or other third party material in this article are included in the article's Creative Commons license, unless indicated otherwise in a credit line to the material. If material is not included in the article's Creative Commons license and your intended use is not permitted by statutory regulation or exceeds the permitted use, you will need to obtain permission directly from the copyright holder. To view a copy of this license, visit http://creativecommons.org/ licenses/by/4.0/.

(C) The Author(s) 2020 